\title{
Research Paper: \\ Effect of Baby Triple P or Positive Parenting Program on Mental Health and Mother-child Relationship in Mothers of Hearing-impaired Children
}

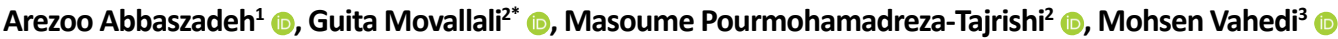

1. Department of Psychology and Exceptional Children Education, Faculty of Behavioral Sciences, University of Social Welfare and Rehabilitation Sciences, Tehran, Iran. 2. Department of Psychology and Exceptional Children Education, Pediatric Neurorehabilitation Research Center, University of Social Welfare and Rehabilitation Sciences, Tehran, Iran.

3. Department of Biostatistics and Epidemiology, Pediatric Neurorehabilitation Research Center, Faculty of Rehabilitation Sciences, University of Social Welfare and Rehabilitation Sciences, Tehran, Iran.

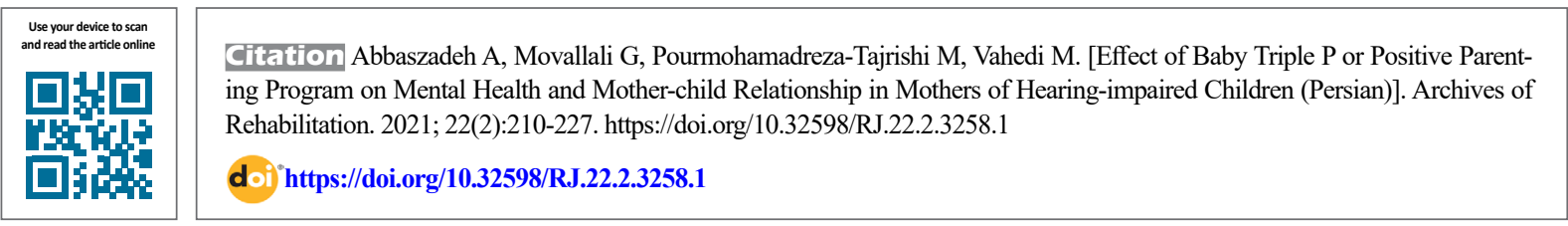

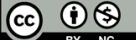

Received: 27 Sep 2020 Accepted: 02 Oct 2020 Available Online: $01 \mathrm{Jul} 2021$

Keywords:

Baby triple P, Hearing loss, Parent-child relationship, Mental Health

\begin{abstract}
Objective Hearing loss in children can affect the parent-child relationship and the mental health of parents, especially mothers as the children's primary caregivers. Maternal mental health problems and challenges in the parent-child relationship can affect the rehabilitation process and the future of hearingimpaired children. Thus, the interventions to promote the parent-child relationship and mental health of parents, especially mothers, should be studied. Triple P or positive parenting program is one of these effective interventional programs. Recently, a new intervention called the Baby Triple P parenting program was developed based on the Triple P program. This study investigates the effect of the Baby Triple $P$ positive parenting program on mental health and mother-child relationship in mothers of children with hearing impairment.

Materials \& Methods This research is a quasi-experimental study with a pretest-posttest design using a control group. The participants were 24 mothers under 3 years old children with hearing impairment referred to the centers for families and children with hearing disabilities in Tehran, Iran, in 2019. They were selected using a convenience sampling method and then randomly divided into groups of intervention $(n=12)$ and control $(n=12)$. The intervention group received the Baby Triple $P$ positive parenting program for 13 training 1-h sessions, one session per week. The control group did not receive any intervention. Both groups completed the mother-child relationship evaluation questionnaire and the symptom checklist-90-revised instrument before and after the intervention. The collected data were analyzed by the Mann-Whitney $U$ test and independent sample t-test.

Results There was no significant difference between the two groups regarding mental health after the intervention $(P>0.05)$. Among four components of the mother-child relationship, the Baby Triple $P$ program had no significant effect on acceptance, overindulgence, and rejection $(P>0.05)$, except on the overprotection component $(\mathrm{P}<0.05)$, which was significantly higher in the intervention group.

Conclusion There is a great need for early interventions for maternal mental health problems and challenges in mother-child relationships for mothers of children with hearing impairment. Although the Baby Triple $P$ is a useful program, it is new for the parents of these children. Considering the unique needs of these children, more studies are needed to enrich the content of this program for the mothers of children with hearing loss to explore its various aspects.
\end{abstract} *Corresponding Author:

Guita Movallali, PhD.

Address: Department of Psychology and Exceptional Children Education, Pediatric Neurorehabilitation Research Center, University of Social Welfare and Rehabilitation Sciences, Tehran, Iran.

Tel: +98 (21) 22180042

E-Mail: drmovallali@gmail.com 


\section{Extended Abstract}

\section{Introduction}

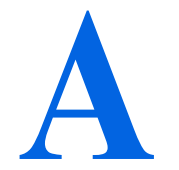

ccording to the 2020 World Health Organization report, there are currently 466 million people in the world with hearing impairment, of whom 34 million are children [1]. Newborn hearing loss has profound effects on the family members and parents [2]. Mothers have an essential and pivotal role in raising their children. So facing any disability of the child, including hearing loss, is more challenging for them, and they are less prepared for it $[3,4]$. Studies on the mental health of mothers of hearingimpaired children have shown that the mental health of these mothers is lower than that of mothers with normal children [5-10]. Maternal mental health problems have several consequences [11-14]. One of the most critical issues affecting mothers' mental health is the parent-child relationship $[15,16]$. A warm, intimate, and trusting relationship between parent and child is an essential factor in child development, and problems in this regard cause problems in the cognitive and emotional-behavioral development of the child [17-20]. Mothers of hearing-impaired children have difficulty building a positive mutual relationship between themselves and their children due to a lack of awareness of the new situation, the nature of the hearing loss, and the resulting psychological stress [21-26]. It is difficult for them to have supportive behaviors, accept the child's disease and provide parenting intimacy and warmth [23]. Since the effects of early interventions are high when parents, especially mothers, are responsive to their child's needs as primary caregivers [27-30], it is necessary to address the emotional dimension of the family and psychological status of mothers in rehabilitation programs along with providing routine services for hearing-impaired children $[9,31]$.

One of the interventions that can be supportive for mothers of hearing-impaired children is Baby Triple P Program. This program is a new intervention that aims to improve the quality of the parent-child relationship and improve the mental health of parents, especially mothers $[32,33]$. It was designed based on level 4 of the positive parenting program. The purpose of this program is to cover the needs of parents of children under 3 years of age who face mental health problems such as anxiety, depression, and stress and are challenged in establishing a relationship with their babies [32]. Studies on the effectiveness of the Baby Triple $P$ Program have shown that this program has been helpful in reducing the mental health problems of parents, especially mothers, and has had a positive effect on the parent-child relationship and the management of behavioral problems in children [33-36]. Because there is a tremendous need for early intervention in Iran that emphasizes the psychological support of parents of hearing-impaired children, this program may be an effective method for hearing-impaired children and their parents. The present study aims to determine the effect of Baby Triple $P$ positive parenting program on mental health and mother-child relationship in mothers of hearing-impaired children.

\section{Materials and Methods}

This research is a quasi-experimental study with a pretestposttest design using a control group. The study population consists of all mothers of hearing-impaired children aged less than 3 years in Tehran Province, Iran, who received services in centers for families and children with hearing disabilities in 2019. After obtaining ethical approval and permissions from the University of Welfare and Rehabilitation Sciences and referring to the clinics, 24 mothers were selected based on the inclusion and exclusion criteria using a convenience sampling method. The mothers were then randomly divided into the intervention and control groups. The inclusion criteria were having a hearing-impaired child under 3 years of age and having at least a high school diploma. The exclusion criteria were having a chronic physical disease, participation in the same intervention simultaneously, and being absent from more than 3 sessions. The mothers in the intervention group received the Baby Triple P program for 13 1-h sessions, one session per week. In both groups, symptom checklist-90-revised was used to measure subjects' mental health, and a Mother-Child Relationship Evaluation (MCRE) questionnaire to measure their motherchild relationship quality at pretest and posttest phases.

The Baby Triple P parenting program has been developed by the Parenting and Family Support Center at the University of Queensland in Australia to enhance the knowledge, skills, and self-confidence of parents who have recently had a baby. Because of the valuable experience of applying the Positive Parenting Program (Triple P) in Iran, correspondence was made with Prof. Sanders, Director of the Parenting and Family Support Center, to obtain his permission to apply to the Baby Triple P parenting program in Iran. Then, due to the cultural differences between Iran and Australia, following the opinion of experts, some changes were made in the way of presenting the contents and in the number of sessions. The main content of the program was adapted from Sanders et al.'s study [33]. To adapt this protocol to the needs of mothers of hearing-impaired children, the resources and workshops that existed in this field were used. Besides, before starting the study, we were in contact with the mothers in one of the centers for families and children with hearing disabilities for 6 months. After collecting data from the subjects, the Shapiro-Wilk test was used to check 
the normality of data distribution. To examine whether the differences between the two study groups were significant, the Mann-Whitney U test was used for the mental health variable and the MCRE questionnaire's component of rejection due to abnormal data distribution. Also, the independent t-test was used for the other three components of acceptance, overprotection, overindulgence due to their normal distribution.

\section{Results}

The mothers in this study had a normal hearing with a Mean \pm SD age of $38.8 \pm 4.55$ years. Also, $70.8 \%$ of them had a high school diploma, $4.2 \%$ had a postgraduate degree, and $25 \%$ had a bachelor's degree. Besides, $20.8 \%$ of them had poor, $50 \%$ moderate, and $29.2 \%$ good socio-economic status. The Mean \pm SD age of their children was $22.13 \pm 1.346$ weeks. All of them were using a hearing aid, except one who had a cochlear implant. The results of the Mann-Whitney U test presented in Table 1 indicate that mental health in the intervention group was not significantly different from that in the control group. Therefore, the Baby Triple P parenting program did not affect the mental health of mothers of hearing-impaired children. The results of the independent t-test (Table 2) indicate that the difference between the two groups was significant only in the overprotection component of the MCRE questionnaire. The differences between the two groups in acceptance and overindulgence components were not significant. Thus, the Baby Triple P parenting program affected only the overprotection component. The results presented in Table 3 indicate that the difference between the two study groups was not significant in terms of the rejection component. Therefore, parenting education did not affect the rejection component, either.

\section{Discussion and Conclusion}

This study aimed to investigate the effect of the Baby Triple $\mathrm{P}$ parenting program on mental health and motherchild relationship in mothers of hearing-impaired children aged less than 3 years. Findings showed that the Baby Triple $\mathrm{P}$ parenting program could not affect the mental health of mothers. It could only affect one of the components of the mother-child relationship (overprotection). Some stud- ies on the effect of the Baby Triple P parenting program on mothers with mental health problems and the parent-child relationship have confirmed the positive effect of this program $[33,34,36]$, while some have not $[34,35]$. Regarding the mental health variable, our results are against the results of some previous studies $[33,34,36]$. One of the possible reasons why the intervention in the present study did not affect mothers' mental health was the novelty of the Baby Triple P program for mothers. This intervention has previously been performed only on mothers of normal-hearing children, and the present study is the first study to evaluate its effectiveness in mothers of hearing-impaired children. The needs and concerns of mothers of normal-hearing and hearing-impaired children are very different. Although efforts have been made to tailor and implement this program based on the needs of mothers of hearing-impaired children, this program was been effective for this group of mothers, and further research is needed. Moreover, mothers in previous studies had children under two years of age or had psychological disorders such as depression, anxiety, and postpartum depression. While in the present study, the age of children ranges from newborn to 3 years, and mothers had no psychological disorders. They were mothers who did not expect the birth of a baby with hearing impairment, and this problem faced them with new challenges.

On the other hand, the insufficient content of the intervention and its low focus on solutions to deal with mental health problems and acceptance of hearing impairment has affected the results. Although the number of intervention sessions was increased, it seems that the content of the sessions needs to be enriched and deepened. Furthermore, one of the most critical issues that should be included in the intervention is accepting the child's hearing loss, because the non-acceptance of a child's hearing loss can be an obstacle to benefiting from psychological interventions [37], and the mothers in this study had a problem in this regard.

Among the mother-child relationship components, the Baby Triple $\mathrm{P}$ parenting program significantly affected the overprotection components. It did not affect acceptance, rejection, and overindulgence components. This result is consistent with the results of some previous studies [34, 35]. Although the Baby Triple $\mathrm{P}$ parenting program is useful and

Table 1. Results of Mann-Whitney U test for mental health variable $(n=12)$

\begin{tabular}{ccccc}
\hline Variable & Group & Median & Interquartile Range & Sig. \\
\hline Mental health & Control & -0.03 & 0.18 & -1.623 \\
& Intervention & -0.38 & 0.105 & 0.79 \\
\hline
\end{tabular}


Table 2. Results of the independent t-test for acceptance, overprotection, overindulgence components of mother-child relationship ( $\mathrm{n}=12)$

\begin{tabular}{|c|c|c|c|c|c|}
\hline Component & Group & Mean \pm SD & df & $\mathbf{t}$ & Sig. \\
\hline \multirow{3}{*}{ Acceptance } & Control & $-1.33 \pm 3.80$ & \multirow{3}{*}{22} & \multirow{3}{*}{-0.459} & \multirow{3}{*}{0.651} \\
\hline & & & & & \\
\hline & Intervention & $-0.33 \pm 6.53$ & & & \\
\hline \multirow{3}{*}{ Overprotection } & Control & $-0.08 \pm 2.39$ & \multirow{3}{*}{22} & \multirow{3}{*}{2.481} & \multirow{3}{*}{0.025} \\
\hline & & & & & \\
\hline & Intervention & $-4.0 \pm 4.92$ & & & \\
\hline \multirow{3}{*}{ Overindulgence } & Control & $-0.50 \pm 3.0$ & \multirow{3}{*}{22} & \multirow{3}{*}{2.028} & \multirow{3}{*}{0.059} \\
\hline & & & & & \\
\hline & Intervention & $-4.33 \pm 5.82$ & & & \\
\hline
\end{tabular}

positively affects the parent-child relationship, its lack of impact on most components of the mother-child relationship in our study may result from mothers' not receiving complete and sufficient information from the intervention. The content of the program needs to be enriched to promote the mother-child relationship and to address more coping strategies for negative emotions such as depression, stress, and anxiety that can affect the quality of the parent-child relationship [34, 35]. Mothers need to learn more about hearing loss, the needs of hearing-impaired children, and how to communicate with them.

It should be noted that the two components of acceptance and rejection are very much affected by the parent's mental health [38]. Since the intervention could not affect mothers' mental health for the reasons stated, the components of acceptance and rejection were not affected. In explaining the ineffectiveness of the intervention on the overindulgence component, the role of the wrong attitude towards and lack of awareness about the hearing loss should also be mentioned. Usually, parents of hearing-impaired children have little information about the hearing loss and the needs of a hearing-impaired child. On the other hand, they believe that since the child is suffering, the child should be allowed to do what $\mathrm{s} /$ he wants $[39,40]$. Mothers in our study have recently found out about their children's hearing loss and had no information about the hearing loss and the needs of their hearing-impaired children. That little information caused the mothers to allow their children to do what they wanted. Finally, it should be noted that the mental health of the parent and the parent-child relationship are highly influenced by social and economic factors [41, 42]. The purpose of this study was not to investigate these factors and control their effects, and their measurement was limited to selfreporting. However, mothers acknowledged the effect of economic and social factors on their attitudes and behaviors in several meetings and referred to them as an obstacle to their management and their relationship with their children, so the effect of these factors cannot be ignored.

There is a high need for early intervention focusing on the mental health and mother-child relationship for mothers of hearing-impaired children. Although the Baby Triple P positive parenting program is useful, further research should be done on it, considering the unique needs of hearing-impaired children aged under 3 years. Also, the program's content should be enriched for the mothers of hearing-impaired children to explore further the various aspects of the program.

\section{Ethical Considerations}

\section{Compliance with ethical guidelines}

Mothers were informed about the study objectives, methods, and duration, and informed consent was obtained. All ethical principles are considered in this article. The participants were informed about the purpose of the research and its implementation stages. They were also assured about the

Table 3. Results of Mann-Whitney U test for the rejection component of mother-child relationship ( $\mathrm{n}=12)$

\begin{tabular}{cccccc}
\hline Component & Group & Median & Interquartile Range & Z & Sig. \\
\hline \multirow{2}{*}{ Rejection } & Control & 0.0001 & 2.50 & & 0.527 \\
& Intervention & 0.50 & 5.75 & & 0.598 \\
\hline
\end{tabular}


confidentiality of their information and were free to leave the study whenever they wished, and if desired, the research results would be available to them.

Funding

This study was extracted from the MA. thesis of the first author at the Department of Psychology and Education of Exceptional Children, University of Social Welfare and Rehabilitation Sciences, Tehran.

Authors' contributions

Conceptualization, editing, and review: Guita Movallali; Data collection: Arezoo Abaszadeh; Methodology: Masoume Pourmohamadreza-Tajrishi; Data analysis: Mohsen Vahedi.

Conflict of interest

The authors declared no conflict of interest. 


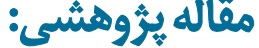 \\ اثربخشى أموزشَ والدَّى برى سالامت روان و رابطه والد كودك در مادران كودكان كمشّنوا

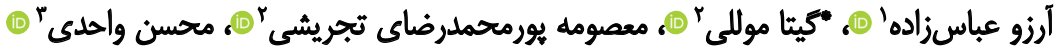 \\ ا. كروه روانشناسي و آموزش كودكان استثنائي، دانشكده علوم رفتارى، دانشعاه علوم توانبخشى و سلامت اجتماعى، تهران، ايران.

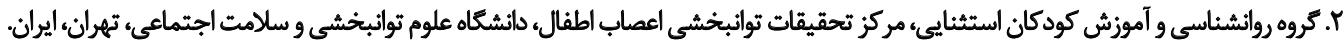

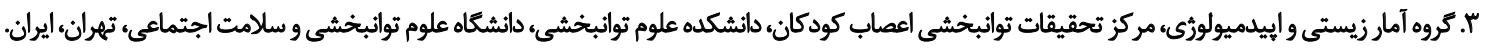

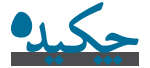

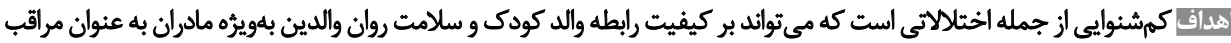

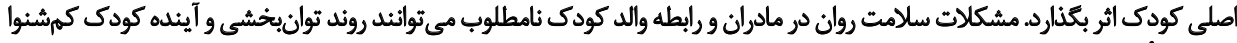

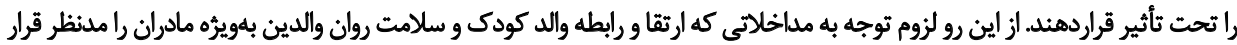

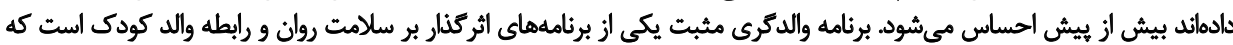

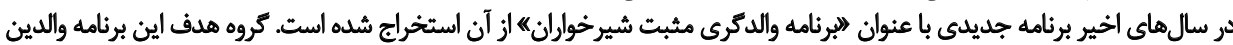

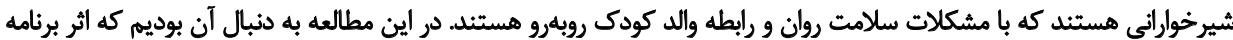

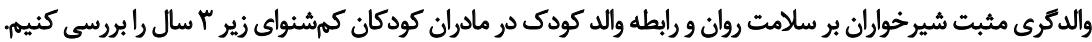

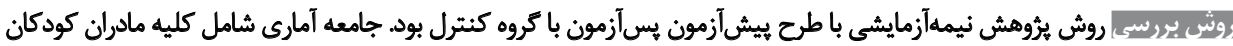

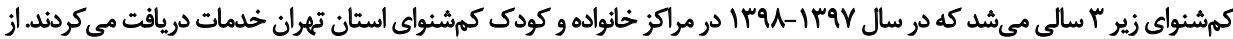

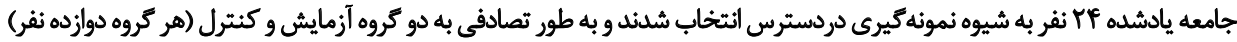

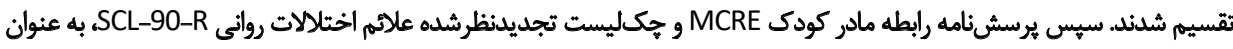

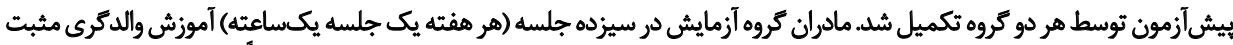

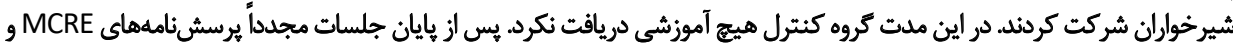

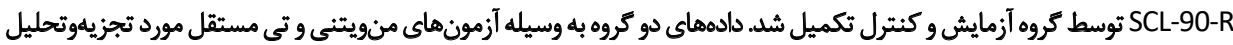

آمارى قرار كرفت.

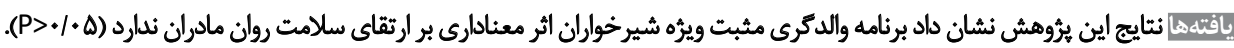

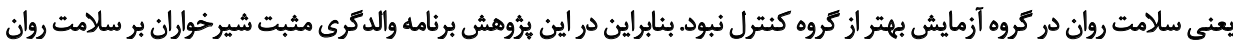

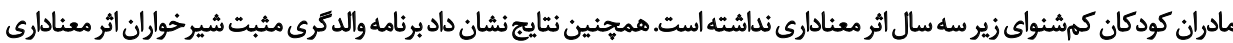

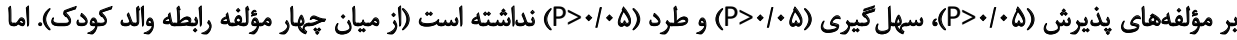

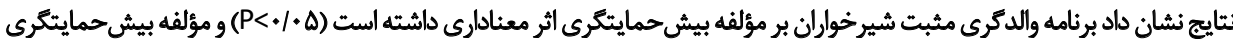

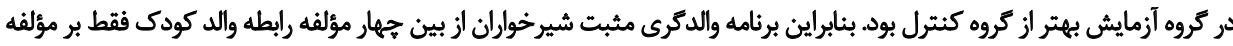

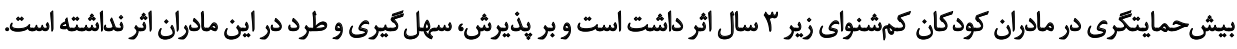

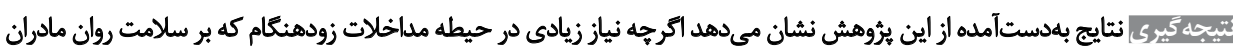

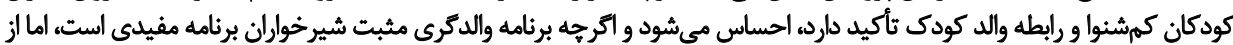

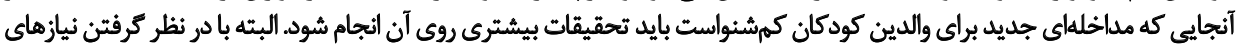

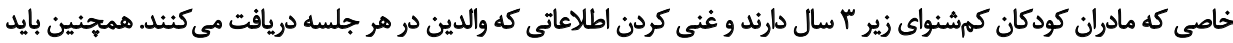

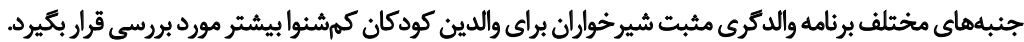

\author{
تاريخ دريافت: 9.0مهر

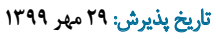 \\ تاريخ انتشار: •ا تير ...
}

-

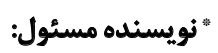

دكتر كيثا موللى نوينده

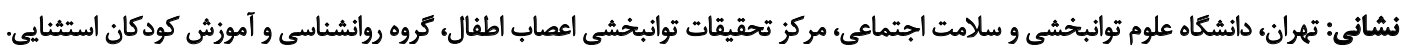

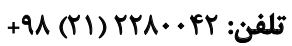

راياثامه: drmovallali@gmail.com 
بيشحمايتخرى، سهل گيرى و طرد فرزند در مادران كودكان با

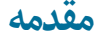

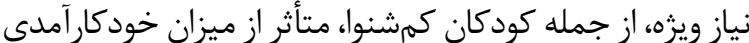

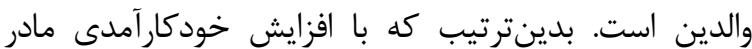

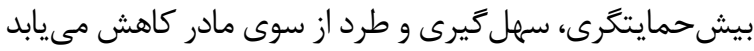

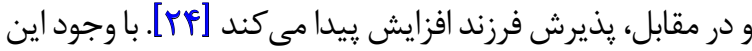

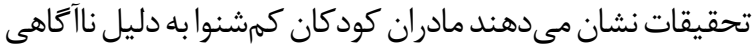

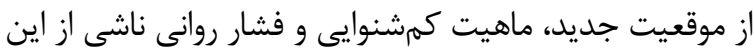

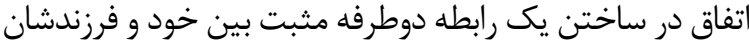

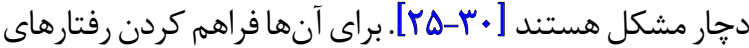

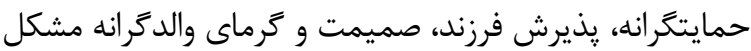

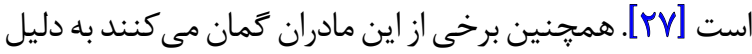

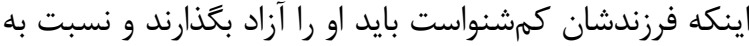

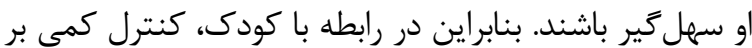

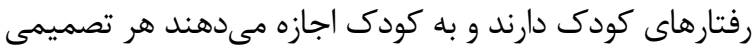

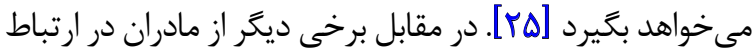

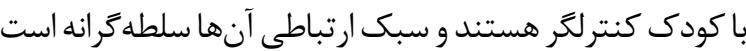

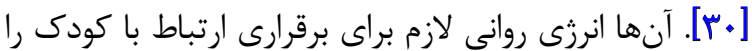

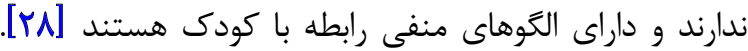

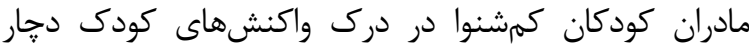

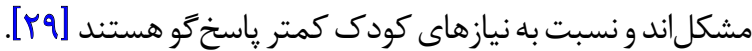

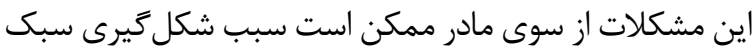

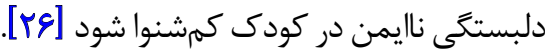

در همين راستا راهنماى مداخلات زودهنكام (بنابر آنجه در اصل

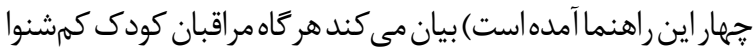

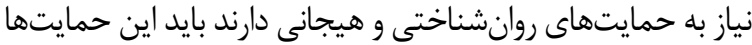

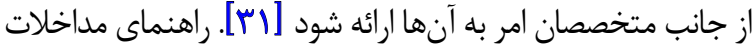

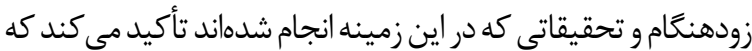

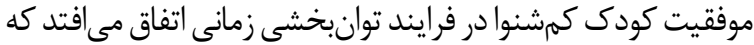

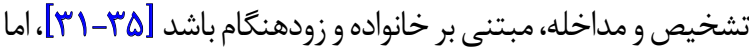

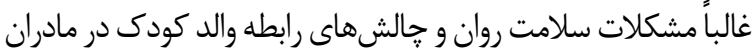

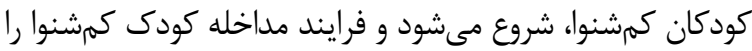

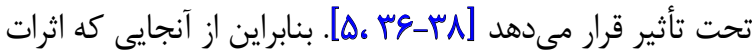

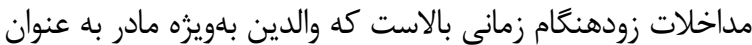

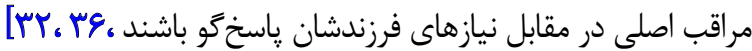

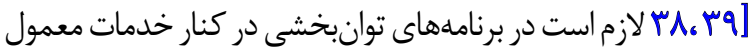

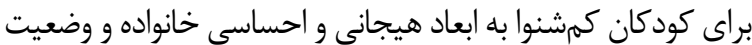

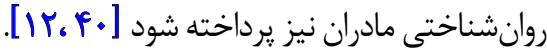

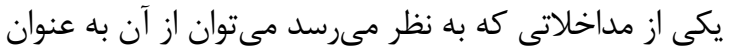

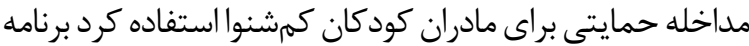

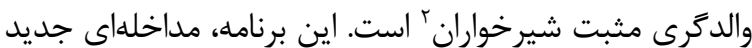

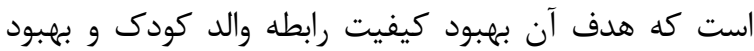

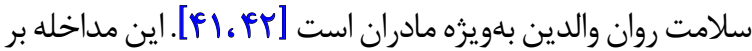

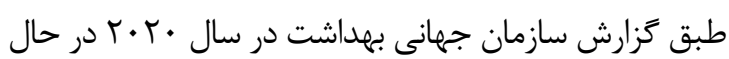

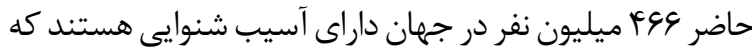

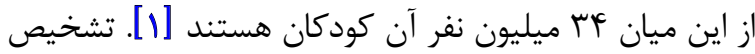

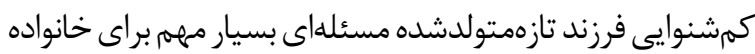

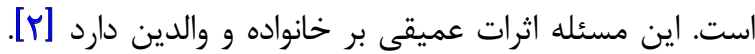

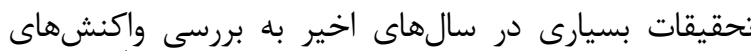

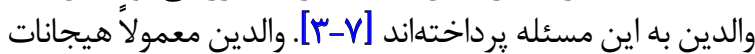

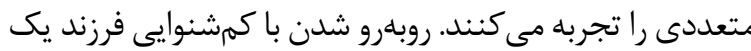

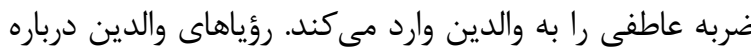

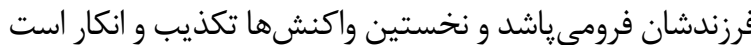

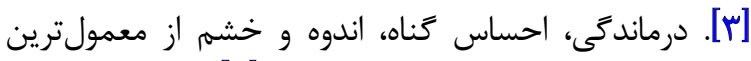

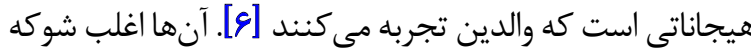

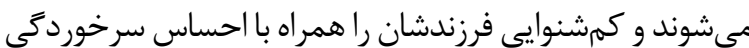

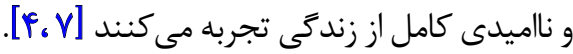

براى مادران به دليل نقش مهام و محورى داى كه در تربيت

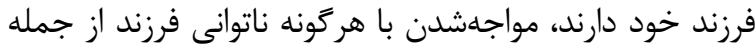

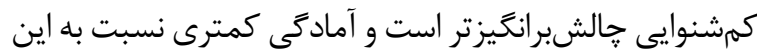

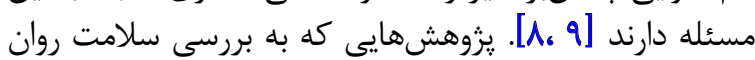

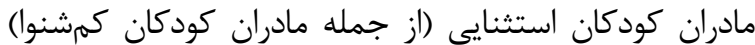

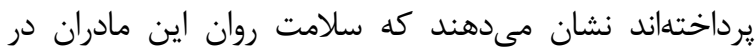

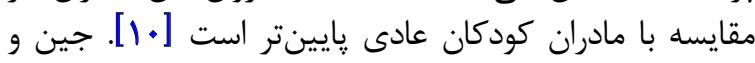

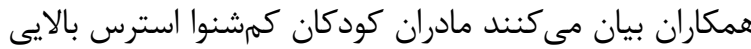

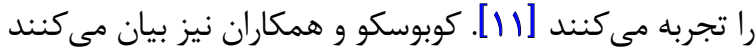

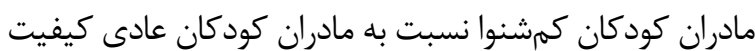

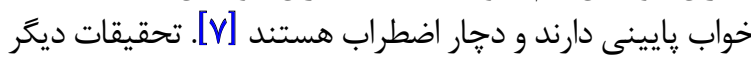

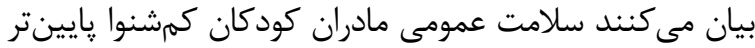

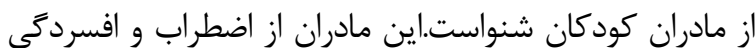

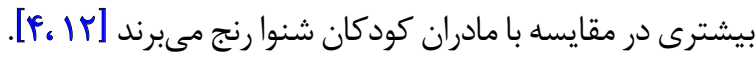

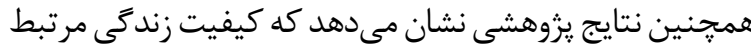

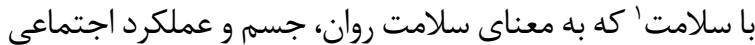

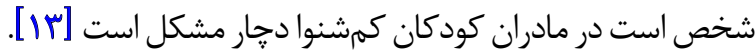

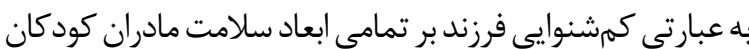

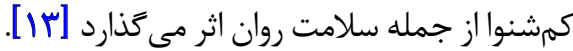

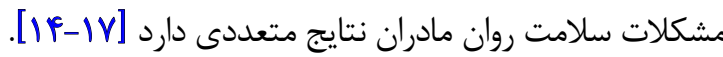

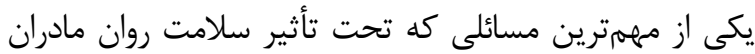

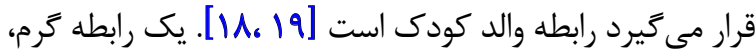

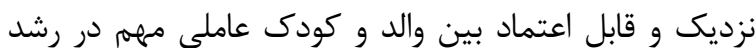

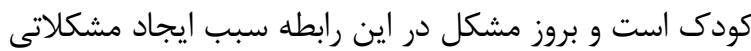

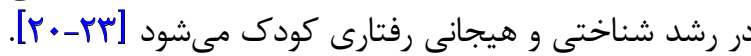

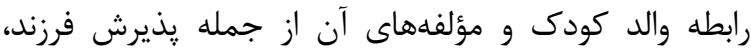


يافتههاى مفيدى در اختيار متخصصان مداخلات زودهنكام قرار

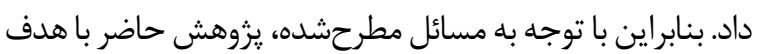

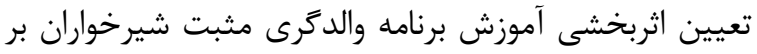

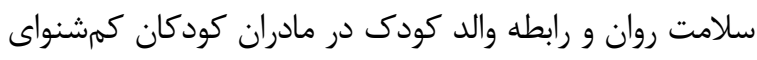

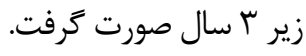

$$
\text { روش بروسى }
$$

اين يزوهش به شيوه نيمهآزمايشى با طرح بيش بيشآزمون

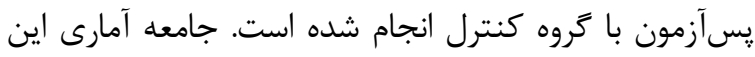

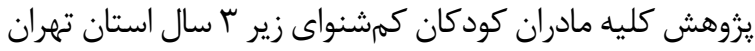

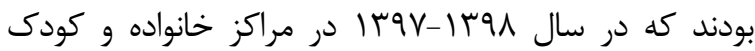

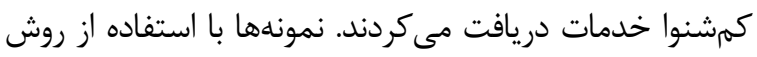

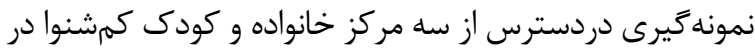

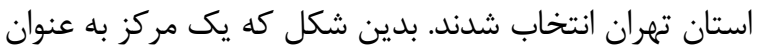

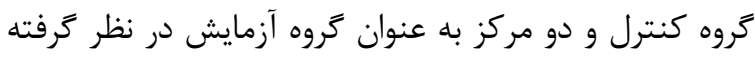

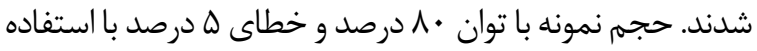

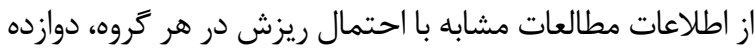

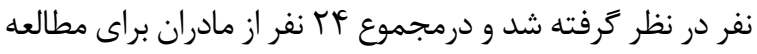

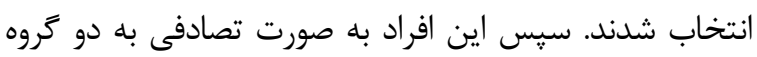

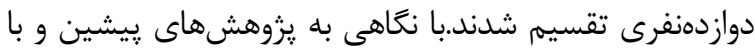

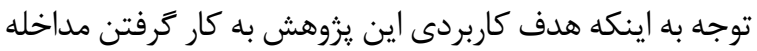

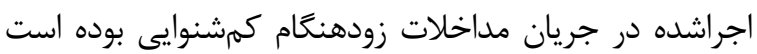

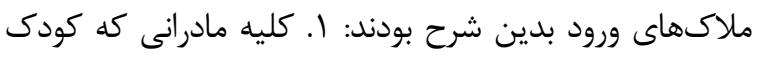

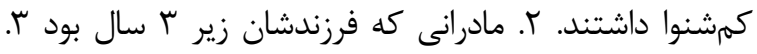

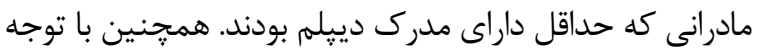

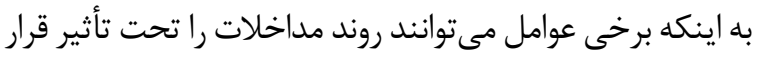

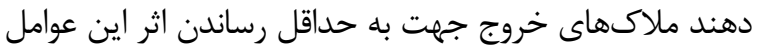

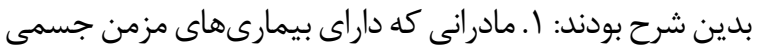

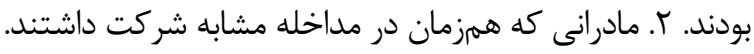

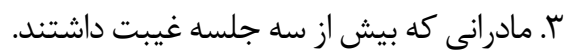
بلمنظور كردآورى دادهاى يزوهش از ابزارهاى زير بهره كرفته

קكىليست تجديدنظرشده علائم اختلالات روانى

فرم اوليه جككليست تجديدنظرشده علائم اختلالات روانى

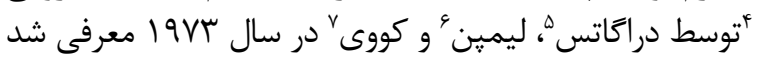

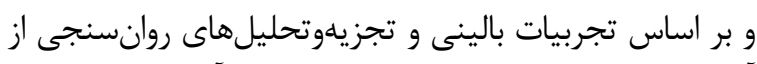

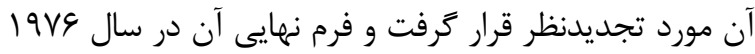

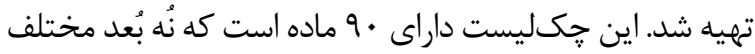

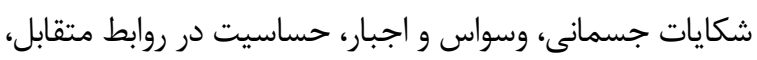

\footnotetext{
4. Symptom Checklist-90-Revised

5. Deragates

6. Limpan

7. Covi
}

بايه سطح جهار برنامه والدگرى مثبت بـ طراحى شده است. هدف

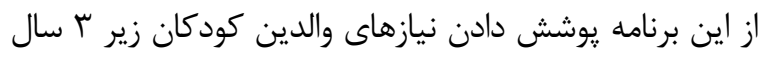

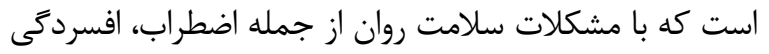

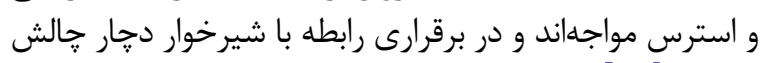

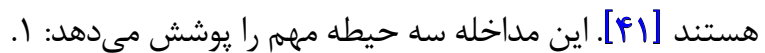

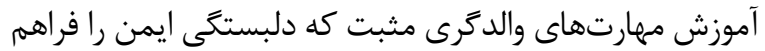

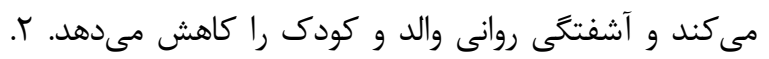

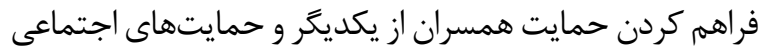

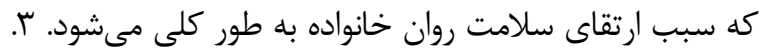

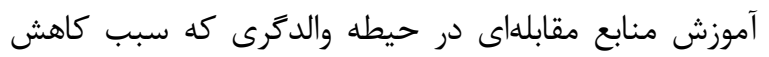

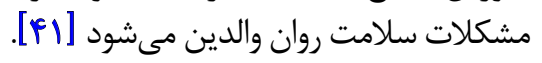

از آنجايى كه برنامه والدكرى مثبت شيرخواران يك مداخله نو

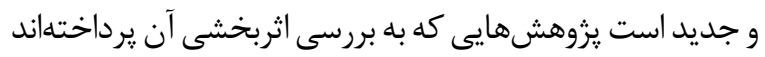

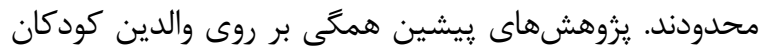

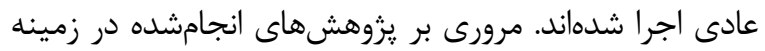

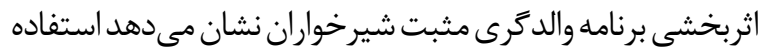

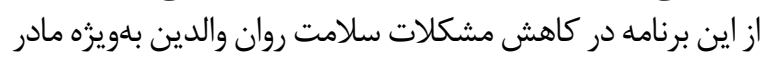

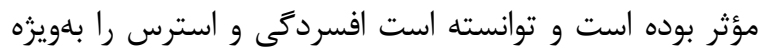
در مادران كاهش دهد [FF-FF_.

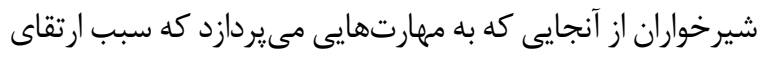

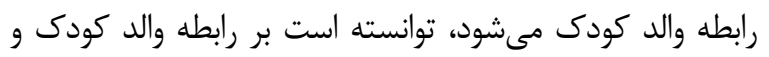

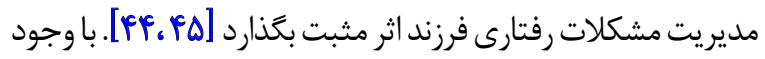

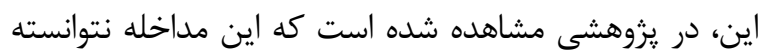

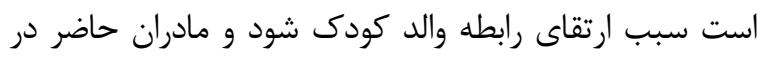

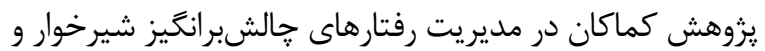

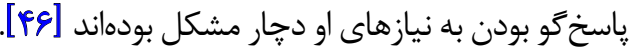

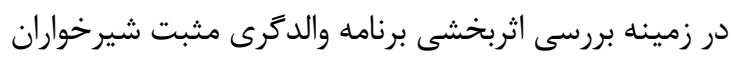

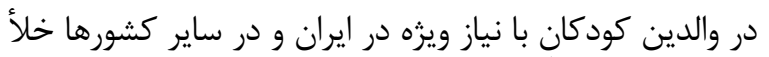

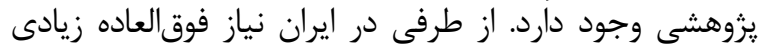

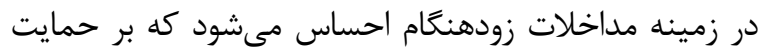

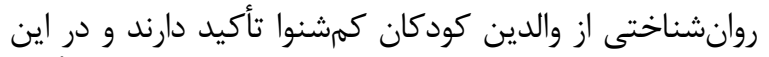

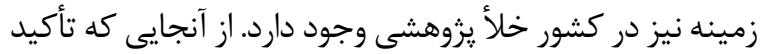

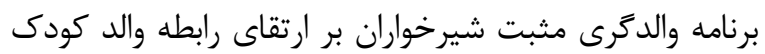

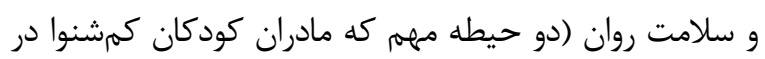

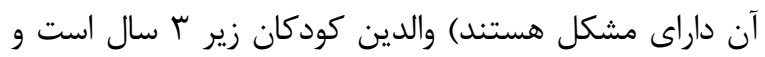

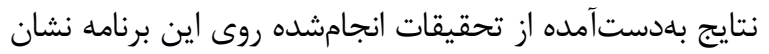

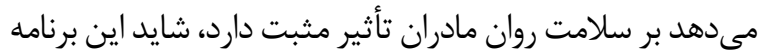

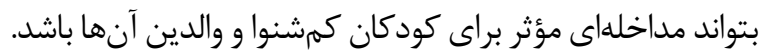

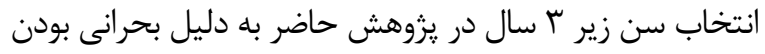

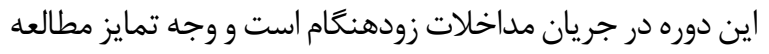

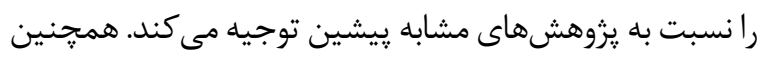

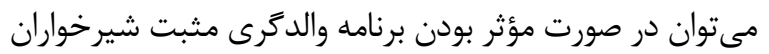

3. Triple $P$ 
برنامه والدَّرى مثبت شيرخواران برنامهوالدكرى مثبت شيرخواران ازمجموعابر نامهاهواى والدكرى

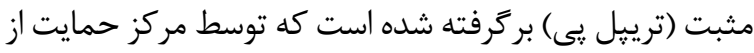

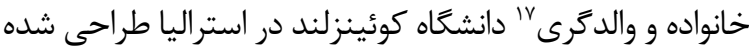

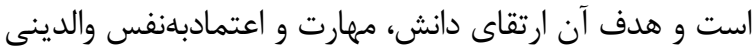

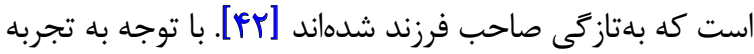

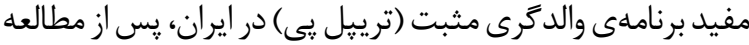

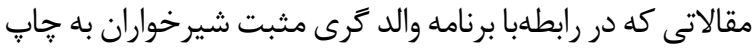

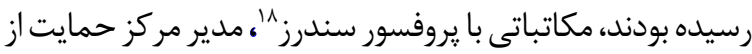

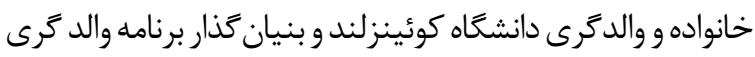

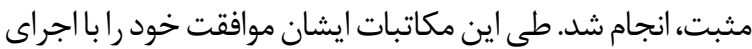

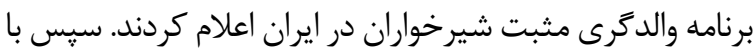

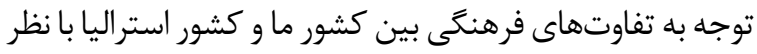

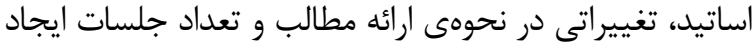

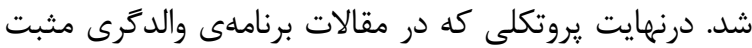

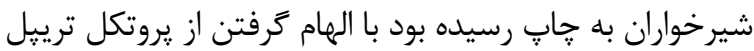

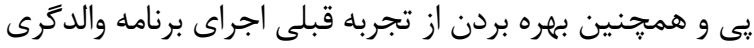

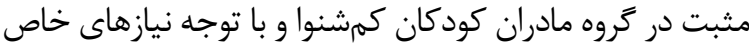

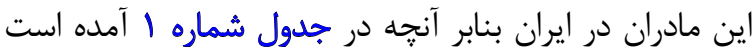

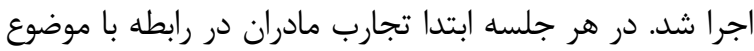

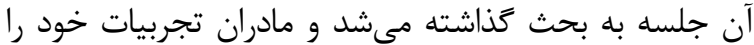

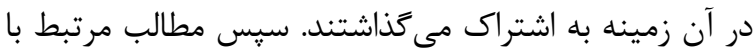

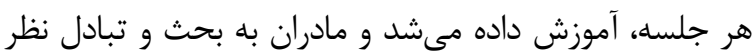

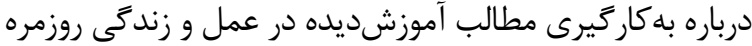

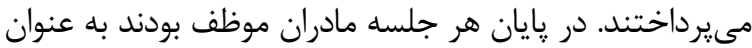

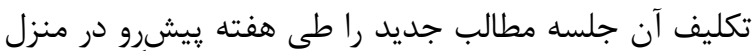

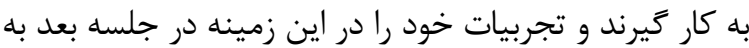

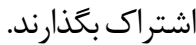

محتواى اوليه و اصلى برنامه والدَّىى مثبت از مقالهاى كه إندار

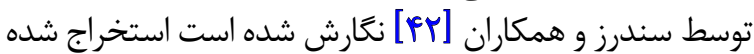

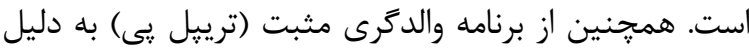

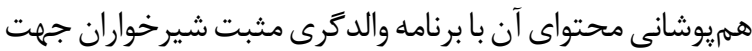

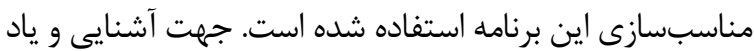

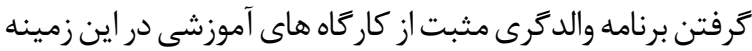

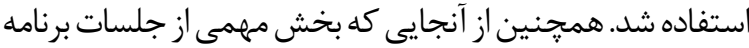

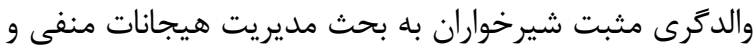

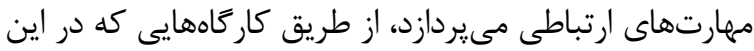

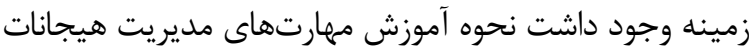

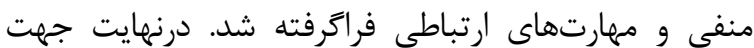

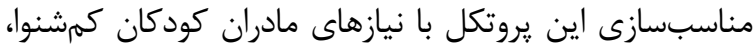

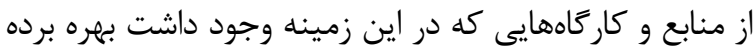

17. Parenting and Family Support Center 18. Matthew R. Sanders

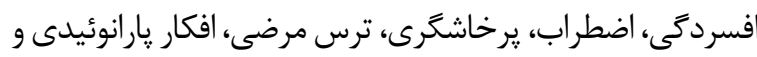

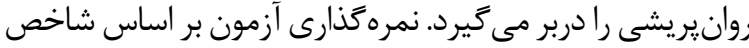

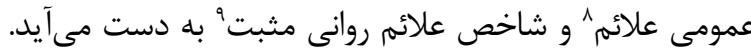

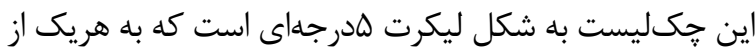

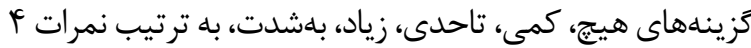

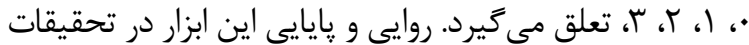

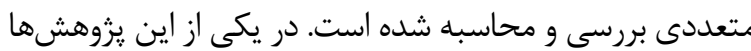

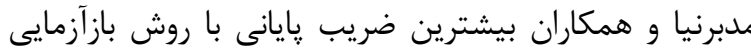

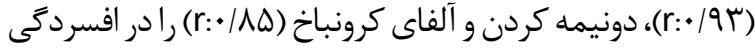

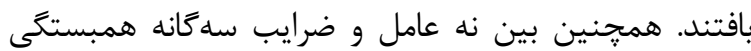

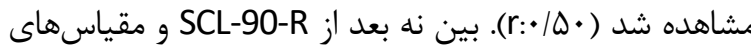

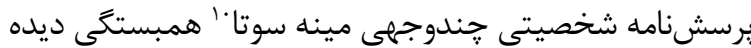

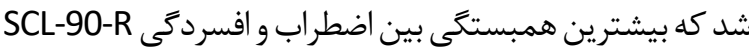
با ضعف روانى در MMPI (r:•/D9) و بين وسواس و و اجبار و و و

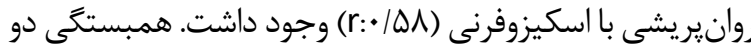
آزمون در سطح هـ • • معنى دار بود.

$$
\text { ير سشنامه ارزيابى رابطه مادر كودى }
$$

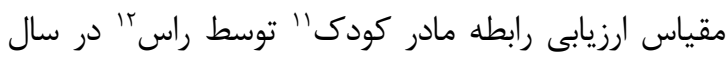

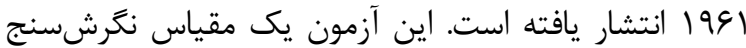

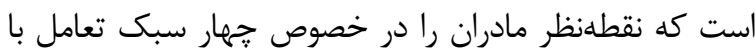

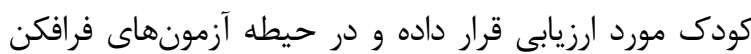

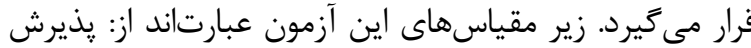

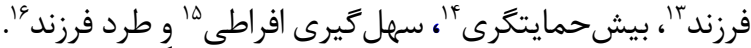

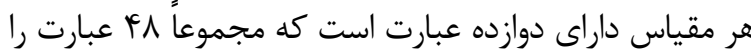

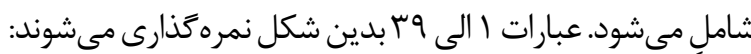

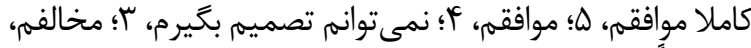

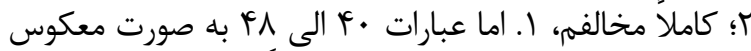

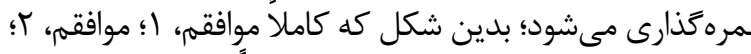

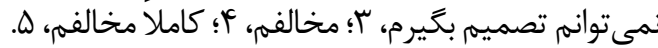

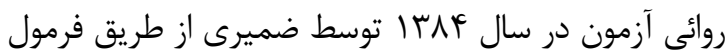

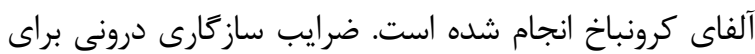

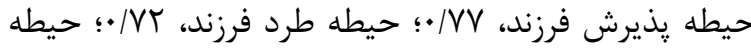

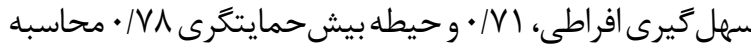

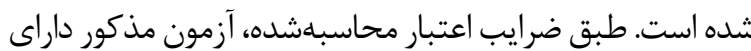

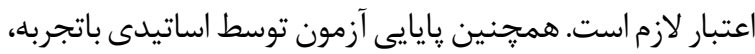
مورد تأييد قرار كرفت و آزمون قابل اجرا دانسته شد.

\section{General Symptom Index}

9. Positive Symptom Distress Index

10. Minnesota Multiphasic Personality Inventory (MMPI)

11. Mother Child Relationship Evaluation (MCRE)

12. Robert

13. Acceptance

14. Overprotection

15. Over indulgence

16. Rejection 


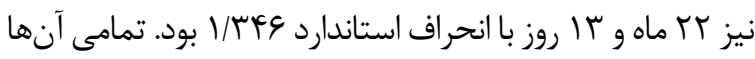

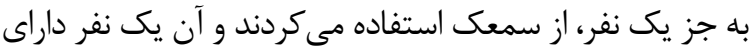
دستخاه كاشت حلزون بود.

در جدول شماره Y شاخصهاى توصيفى متغيرهاى سلامت

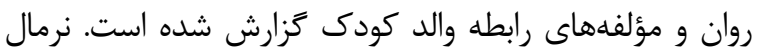

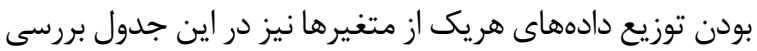

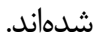

نتايج ارائهشده در جدول شماره Y بيانكر آن است كه

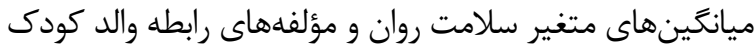

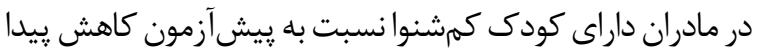

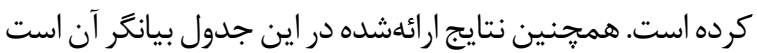

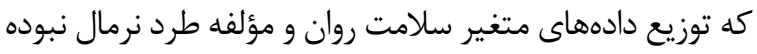

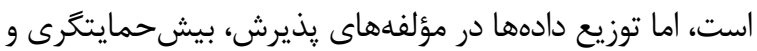

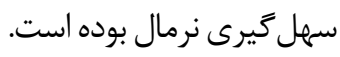

بدين ترتيب يس إز تعيين نمرات افتراقى، براى بررسى اينكه

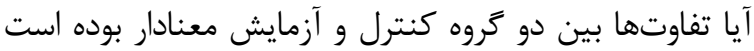

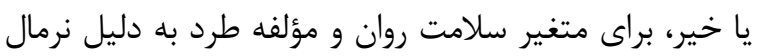

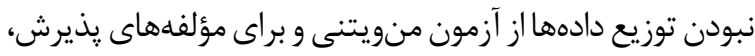

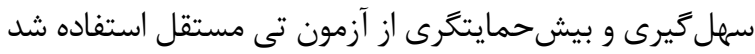

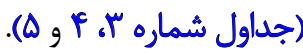

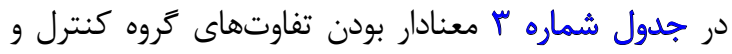

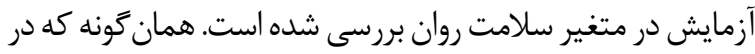

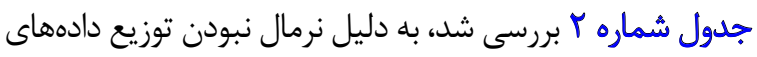

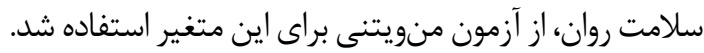

نتايج ارائهشده در جدول شماره ب بيانكر آن است كه سلامت

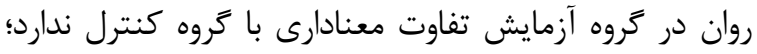

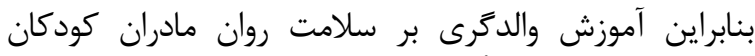
كمشنواى زير ب سال تأثير نداشته است

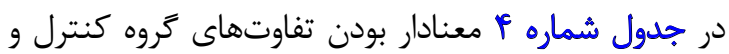

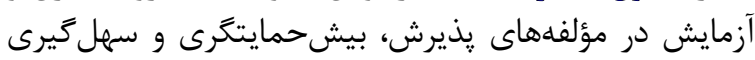

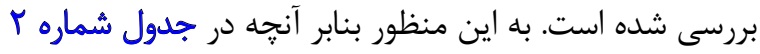
كزارش شده است به دليل نرمال نبودن توزيع دادهها از آزمون تى مستقل استفاده شد.

نتايج ارائهشده در جدول شماره F أبيانكر آن است كه تفاوت

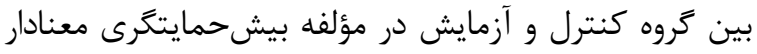

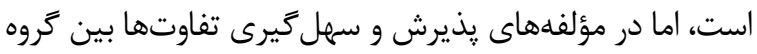

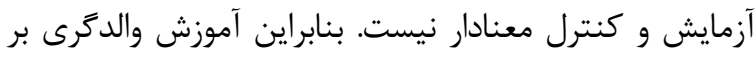

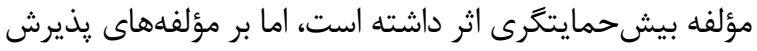

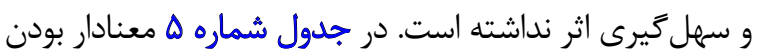

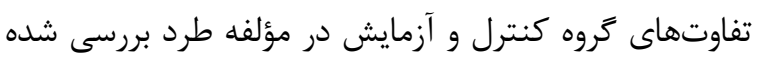

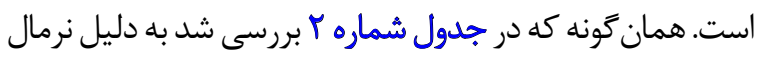

شد. همجنين ييش از شروع مداخله و براى غنى كردن اطلاعات

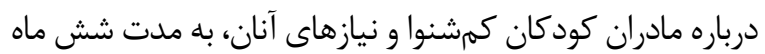

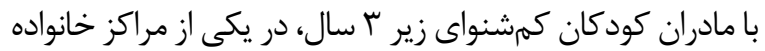

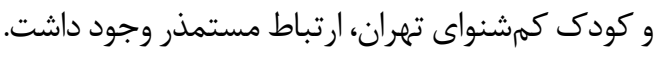

$$
\text { روش بررسى }
$$

در ابتدا يس از دريافت كد اخلاق از كميته اخلاق دانشحاه

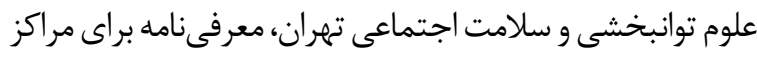

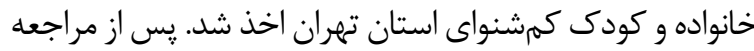

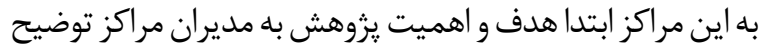

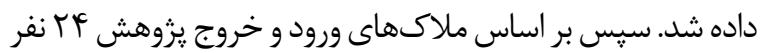

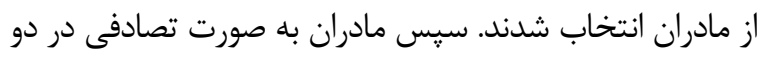

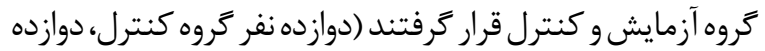

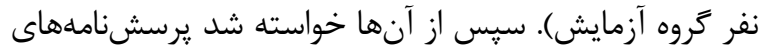

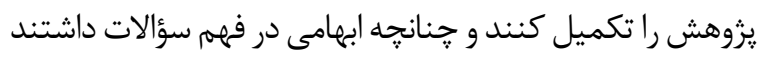

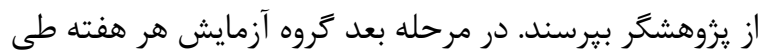

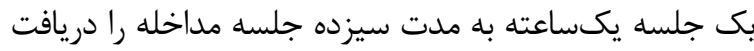

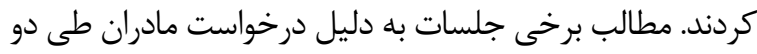

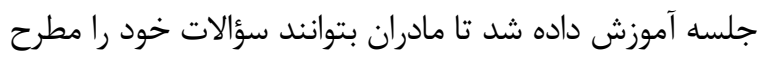

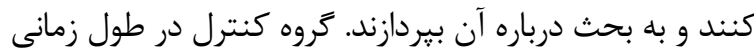

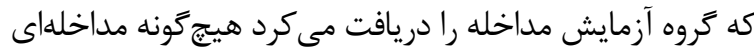

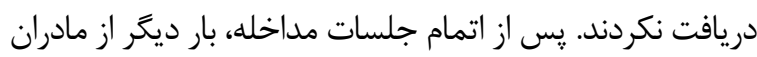

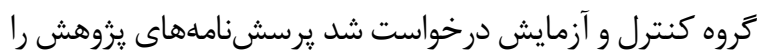

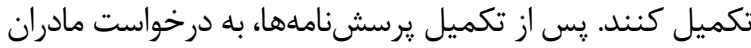

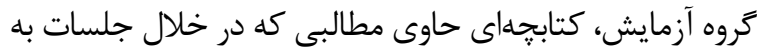

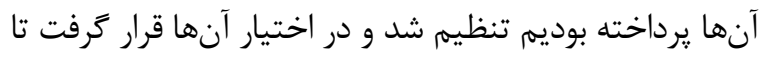

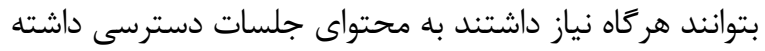

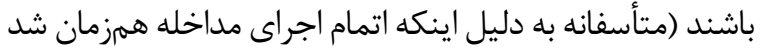

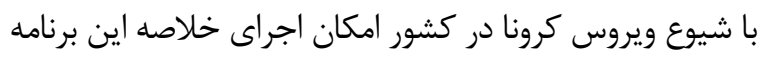

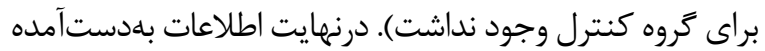

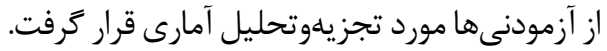

يافتهها

ابتدا شاخصهاى توصيفى از قبيل درصد، ميانگين و انحراف

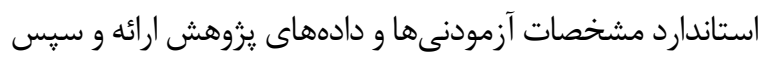

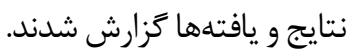

مادرانى كه در اين يزوهش حضور داشتند همكى شنوا بون بودند

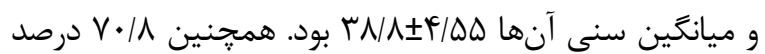

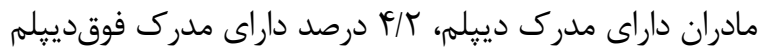

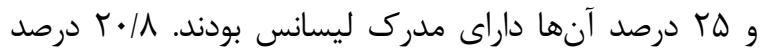

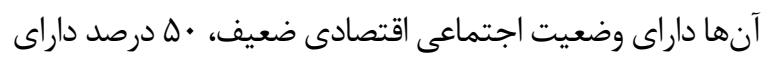

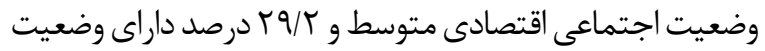

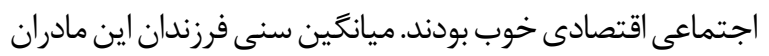


جدول ا. يروتكل برئامه والدترى مثبت شيرخواران

\begin{tabular}{|c|c|c|c|}
\hline إهبردها & اهداف & 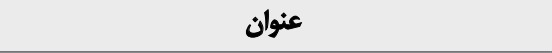 & 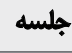 \\
\hline برسى الجمالى جلسات ييشرو & آماده كردن مادران براى جلسات والدكرى مثبت & 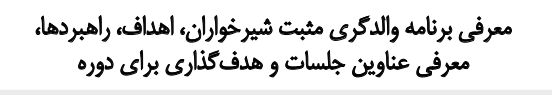 & 1 \\
\hline 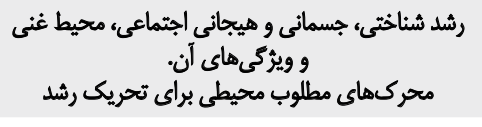 & ارتقاى رابطه والد كودى & معرفى مراحل رشدى شيرخوار و عوامل اثركنار بر آن & r \\
\hline 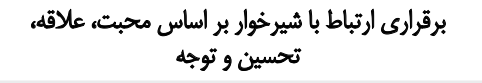 & ارتقاى رابطه والد كودى & معرفى راهكارهايع براي ياسخ دادن به نوزاد & $r$ \\
\hline 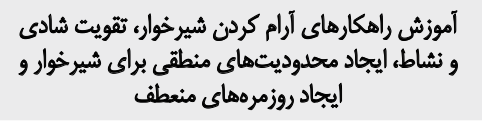 & برقرارى ارتباط با شيرخوار متثاسب با سن او و & 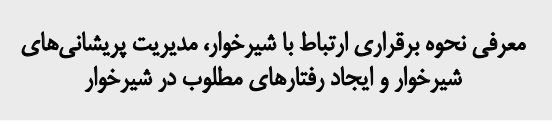 & $\varphi$ \\
\hline حمايت اجتماعى & هديريت موقعيتهاى جالشبرانكيز & معرفى تجارب مشترى والدين و خطاها و تلههاى رائج والدگىى & $\Delta$ \\
\hline ناديده كرفتن، يرت كردن حواس، قاطعانه برخورد كردن & مديريت موقعيتهاى جالشبرانكيز & آموزش راهكارهاى مقابله با بدرفتارى در شيرخواران & 8 \\
\hline مديريت السترس، مديريت خُشهي، مهارت برقرارى ارتباطة & 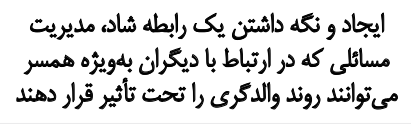 & معرقى هيجانات منفى و مشكلات الرتباطيى با همسر و اثرات & $V-I r$ \\
\hline تقويت خودتنظيمى و هدف كذارى براى آيثنه & نكادها|شتن تغييرات & جمعبندى و به الشتراى كناششن تجربيات & ir \\
\hline
\end{tabular}

نبودن توزيع دادههاى مؤلفه طرد، از آزمون منويتنى براى اين بحث هدف اين :زروهش مطالعه تأثير آموزش والدگرى بر سلامت

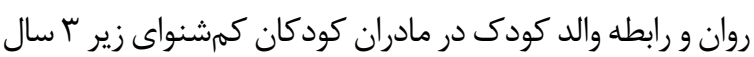

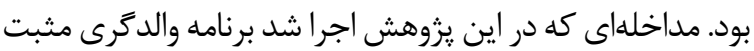

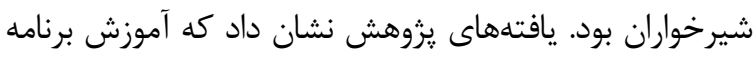

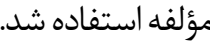

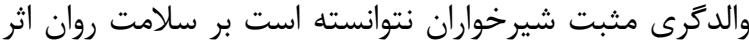

نتايج ارائدشده در جدول شماره ه بيانكَر آن است كه تفاوت

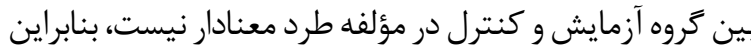
آموزش والدگرى بر مؤلفه طرد اثر نداشته است.

جدول I. شاخصهاي توصيفى متغيرهاي يُروهش

\begin{tabular}{|c|c|c|c|c|c|c|c|c|c|}
\hline \multicolumn{4}{|c|}{ يسآزمون } & \multicolumn{4}{|c|}{ يشي آزمون } & \multirow{2}{*}{ تمروه } & \multirow{2}{*}{ مثغير } \\
\hline $\mathbf{P}$ & $\mathbf{Z}$ & ميانكين+|نحراف|ستائدارد & فراوانى & $\mathbf{P}$ & $\mathbf{Z}$ & ميانكين+|نحراف|ستاندارد & فراوانى & & \\
\hline $.1 . W$ & IAF & $\cdot M^{2} \cdot \pm \cdot M \cdot r$ & $\pi$ & $\therefore / .49$ & INET & ./AGYI./TIA & r & كثترل & \\
\hline.$/ 18$ & $. / 9 .$. & $\cdot / \Delta A Y \pm+/ I F$ & ir & - TreV &.$/ 911$ & $V \cdot r \pm \cdot / r \Delta \Delta$ & ir & آزمايش & سلامت روان \\
\hline.$/ M M$ &.$/ 9 \Delta \Delta$ & $r q / q T \pm 1 / e q$ & ir & . /arr &.$/ 94$ & $F \mid / T \Delta \pm 1 / \mu \varphi$ & $\pi$ & كترل & \\
\hline$+/ M e q$ &.$/ 941$ & $F \cdot / A \Psi \pm V / A I$ & $\pi$ & $+/ 999$ &.$/ 499$ & $\varphi v / I V \pm V / / q 9$ & ir & أزمايش & ليذيرش \\
\hline $1+\pi r$ & INEA & $T S / \cdot A \pm T / T r$ & $\pi$ & $+/ T+8$ &.$/ 9.9$ & $r \& / I V \pm r / / Q$ & ir & كنترل & \\
\hline.$/ 1 n$ & .9 .4 & $n / q Y \pm T / .8$ & $\pi$ &.$/ 9 r^{n}$ &.$/ 9 \vee A$ & TNAYAY/TI & ir & أزمايش & \\
\hline$. / 4+1$ &.$/ 9 \pi$ & $T / / T \Delta \pm Y / \odot 1$ & $\pi$ &.$/ T H$ &.$/ 914$ & $r V / V \Delta \pm V / F V$ & rr & كثترل & \\
\hline.$/ 291$ &.$/ 919$ & $r \Delta / 9 Y \pm 1 / M C$ & ir &.$/ 1 A F$ &.$/ 199$ & $\varphi \cdot / r \Delta \pm 1 / \Lambda$. & ir & آزمايش & سهل كيرى \\
\hline.$/ 911$ &.$/ 9 \%$ & YQ/AYI./9YA & ir &.$/ M$ &.$|A M|$ & $\Gamma \Delta / V \Delta \pm 1 / T A$ & ir & كتترل & \\
\hline קו & . /aTr & $\Gamma \Delta / T \Psi 1 / 1 \Delta$ & $\pi$ & $+1++1$ & . Mar & $r \Delta / T \Delta \pm 1 / 9 T$ & $\pi$ & أزمايش & \\
\hline
\end{tabular}


جدول ب. نتايج آزمون منويتنى براى متغير سلامت روان

\begin{tabular}{|c|c|c|c|c|c|c|}
\hline مقدار احتمال & أماره & دامثه ميان هاركى & ميانه & تعداد & تمروه & متغير \\
\hline \multirow[b]{2}{*}{$\cdot N=\Delta$} & \multirow[b]{2}{*}{$-1 / g \pi T$} &.$M A$ &.$- \%$ & r & كنترل & \multirow[b]{2}{*}{ سلامت روان } \\
\hline & &. $\mathrm{ma}$ &.$- / 4 a$ & $i r$ & آزمايش & \\
\hline
\end{tabular}

مادران كودكان كمشنوا متناسبسازى و اجرا شود، اين برنامه در

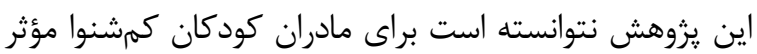

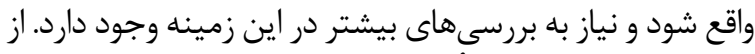

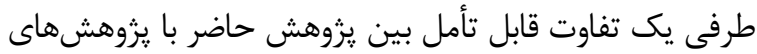

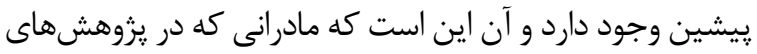

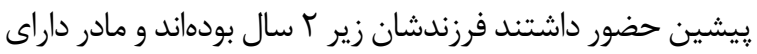

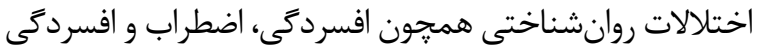

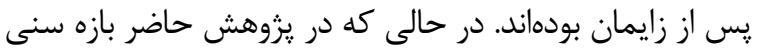

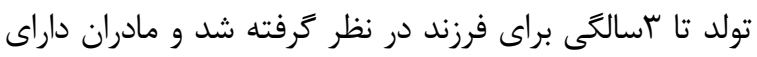

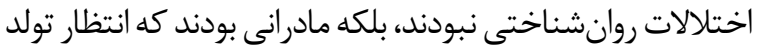

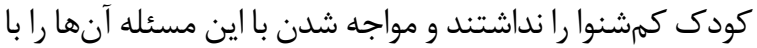
جالشهاى جديدى روبهرو كرده بود.

از طرفى به نظر مى رسد كافى نبودن محتواى مداخله و تمركز

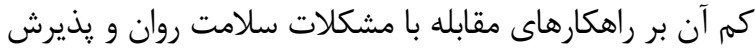

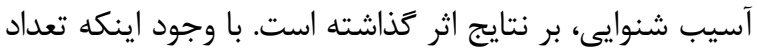

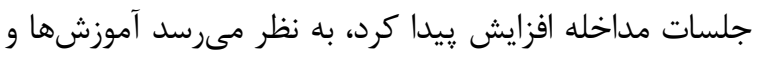

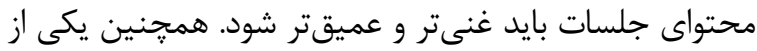

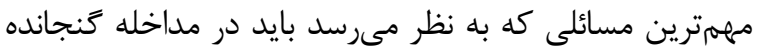

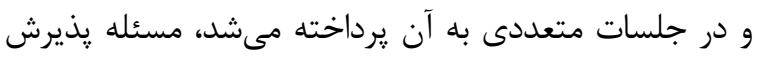

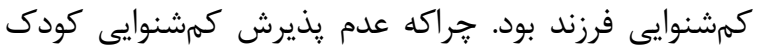

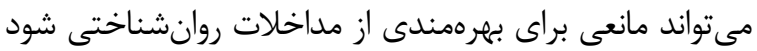

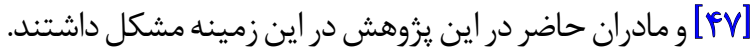

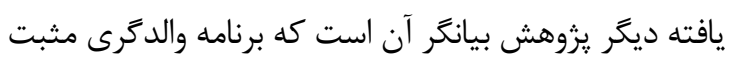

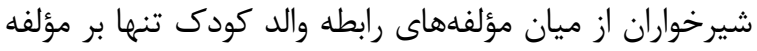
بيشحمايتخرى اثر داشته است و نتوانسته است بر مؤلفي مؤلفههاى
بحذارد و تنها توانسته است بر يكى از مؤلفههاى رابطه والد كودى

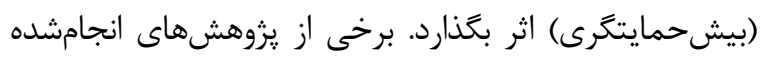

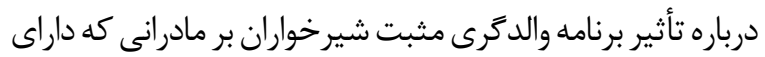

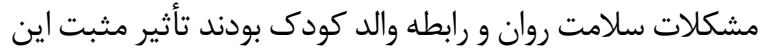

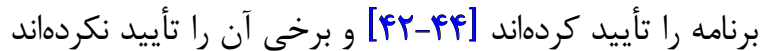

[ [Fa, pq]

نخستين يافته يزوهش نشان داد استفاده از برنامه والدَّىى

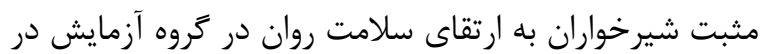

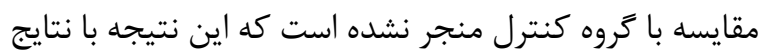

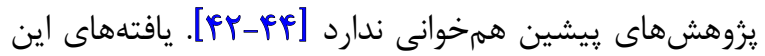

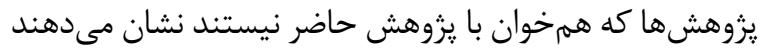

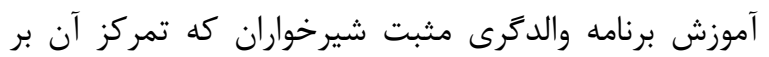

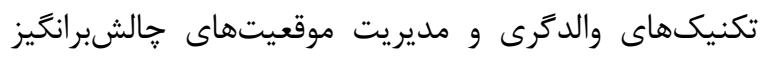

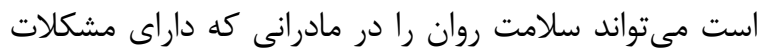

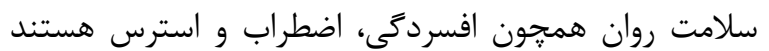

ارتقا دهد [Fr-FF]

يكى از اولين مسائلى كه مىتوان نقش آن را در عدم اثر كذارى

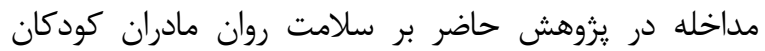

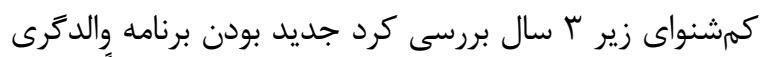

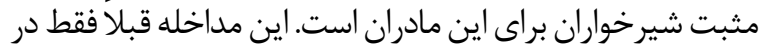

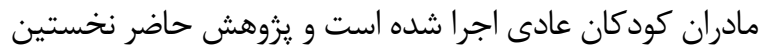

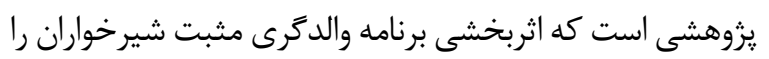

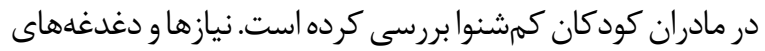

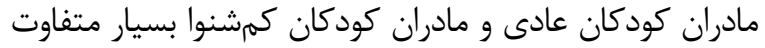
است و باوجود اينكه تلاش شده بود اين يروتكل بر اساس نياز هان مانى

جدول ع. نتايج آزمون تى مسيّقل براى مؤلفههاى بذيرش، بيش حمايتُرى و سهل كيرى

\begin{tabular}{|c|c|c|c|c|c|c|}
\hline مقدار احتمال & t & درجه أزادى & ميانكيندانحراف استاندارد & تعداد & كروه & 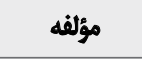 \\
\hline \multirow[b]{2}{*}{.$|F \Delta|$} & \multirow[b]{2}{*}{$-0 / 509$} & \multirow[b]{2}{*}{ rr } & $-I / / \Psi \pm \Psi / A$. & ir & كتترل & \multirow[b]{2}{*}{ يذيرش } \\
\hline & & & & & & \\
\hline \multirow[b]{2}{*}{$.1+50$} & \multirow[b]{2}{*}{$r / F A I$} & \multirow[b]{2}{*}{ tr } & $-. / .1 \pm 7 / \% 9$ & ir & 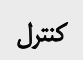 & \multirow[b]{2}{*}{ بيشحمايثكرى } \\
\hline & & & $-r \pm r / q r$ & ir & آزمايش & \\
\hline \multirow[b]{2}{*}{.1 .09} & \multirow[b]{2}{*}{$T / \cdot Y \Lambda$} & \multirow[b]{2}{*}{ r } & $-\cdot / \Delta \cdot \pm r$ & $r$ & كتترل & \multirow[b]{2}{*}{ سهلكيرى } \\
\hline & & & $-P / R T \pm \Delta / A T$ & ir & أزمايش & \\
\hline
\end{tabular}


جدول ه. نتايج آزمون منويتنى براى مؤلفه طرد

\begin{tabular}{|c|c|c|c|c|c|c|}
\hline مقدار احتمال & آماره & دامثه ميان هاركى & مياته & تعداد & كروه & متغير \\
\hline \multirow{2}{*}{.$/ \Delta 4$} & \multirow{2}{*}{. lorr } & $\Gamma / \Delta$. & $\% \ldots$ & ir & كتثرل & \multirow{2}{*}{ طرد } \\
\hline & & $\Delta / v \Delta$ &.$/ \Delta$ & Ir & آزمايش & \\
\hline
\end{tabular}

بوده است، اما از آنجايى كه در جلسات متعدد مادران اذعان

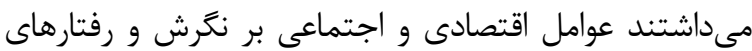

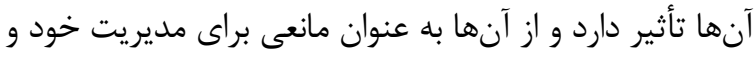

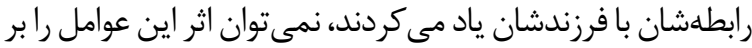

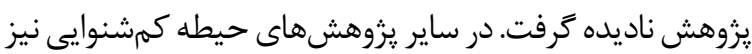

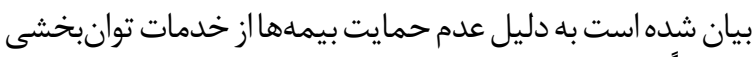

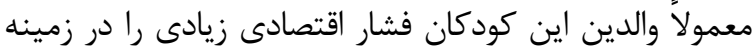

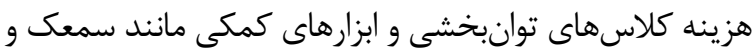

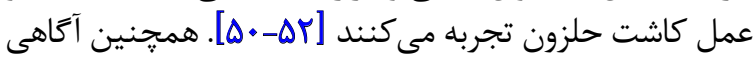

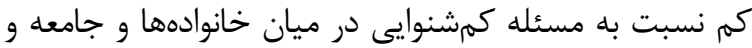

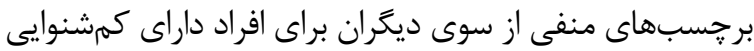

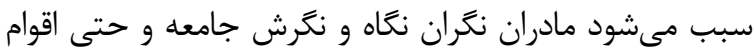

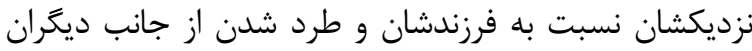

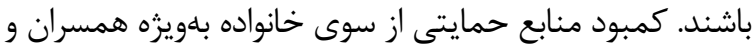

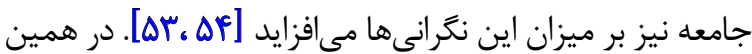

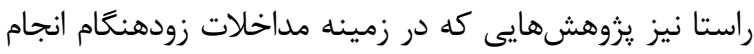

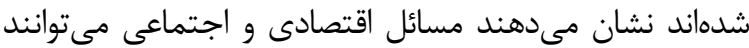

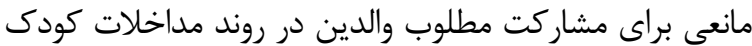

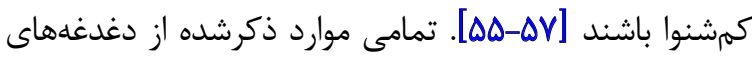

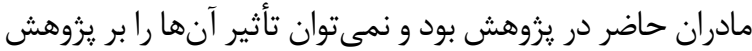

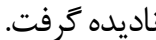

\section{نتيجهليرى}

نتايج بهدستآمده از اين يزوهش نشان مى دهد اتر جها برنامه

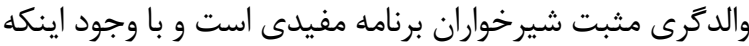

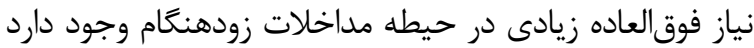

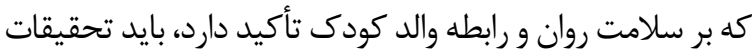

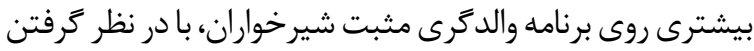

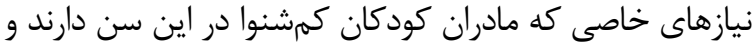

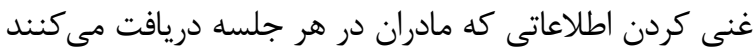

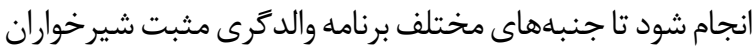

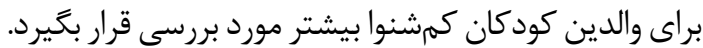
هماند هر يزوهش ديخرى، يزوهش حاضر نيز با

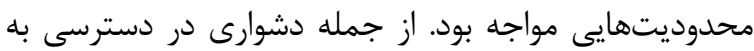

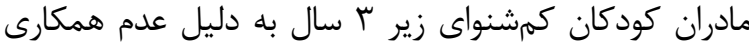

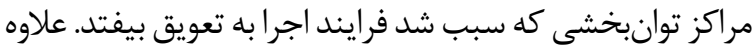

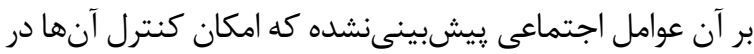

يذيرش، طرد و سهل گيرى اثر بخذارد. اين نتيجه با نتايج برخى إنى إنى

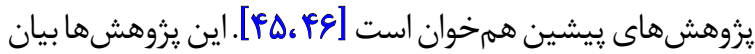

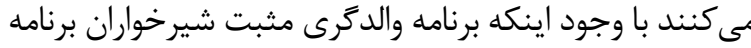

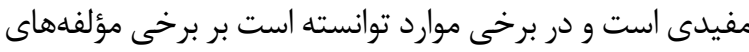

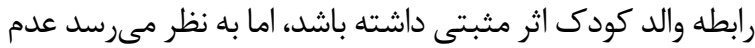

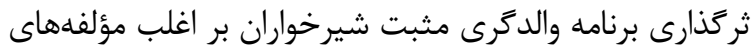

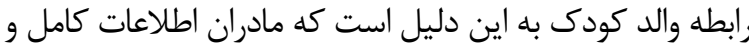

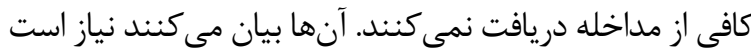

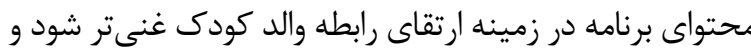

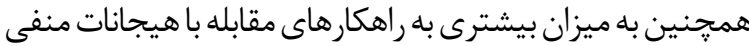

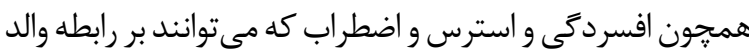
كودى اثربخذارند، يرداخته شود [هF-fF]

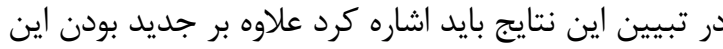

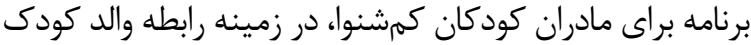

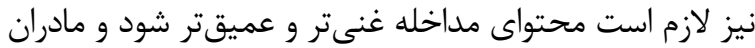

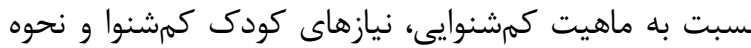

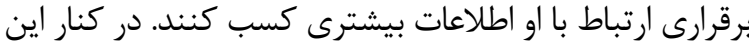

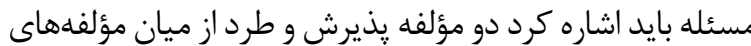

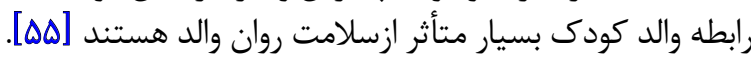

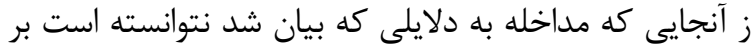

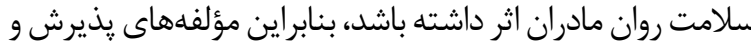

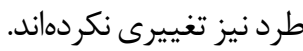
در تبيين عدم اثر گذارى مداخله بر مؤلفه سهل كيرى نيز بايد به

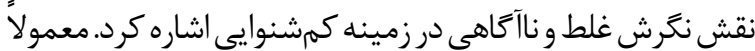

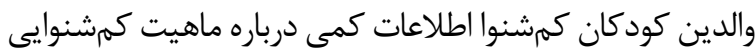

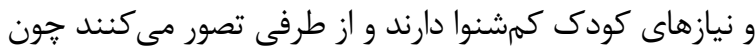

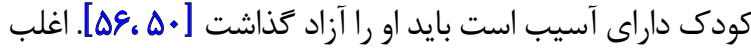

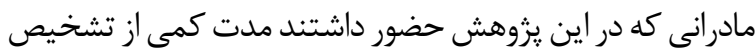

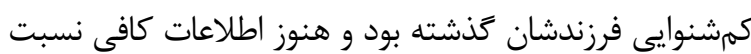

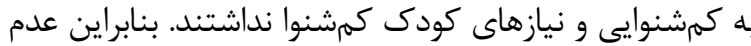

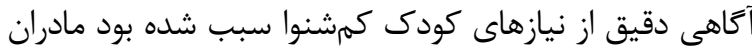
كودى را آزاد بكذارند و سمل كئرير باشند.

سلامت روان والد و رابطه والد كودى بسيار تحت تأثير عوامل

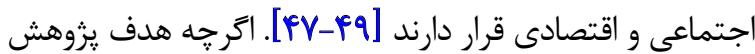

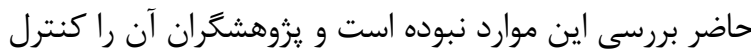

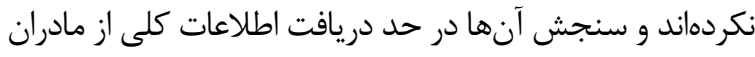


همه شركت كنند مان در جريان روند يزوهش بودند. اطلاعات آن

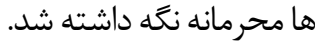

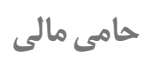

اين مقاله از קاياننامه كارشناسى ارشد نويسنده اول كروها

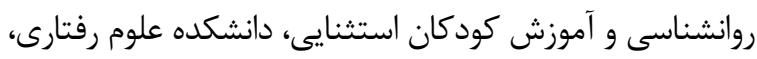
دانشكاه علوم توانبخشى و سلامت اجتماعى، تهر انئ استان.

$$
\text { مشاركت نويسندكان }
$$

مفهومسازى، ويراستارى و نهايىسازى مقاله: گَيتا موللى؛

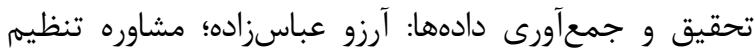

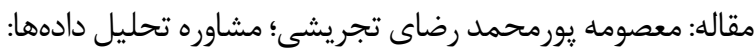

$$
\text { تعارض محن واحدى. }
$$

بنا بر اظهار نويسند
زمان اجراى مداخله ممكن نبود بر روند مداخله اثر كذاشتند. از

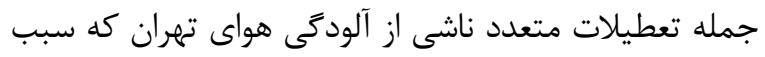

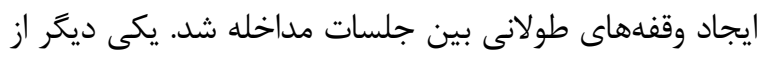

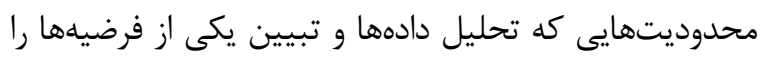

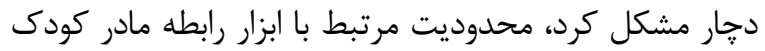

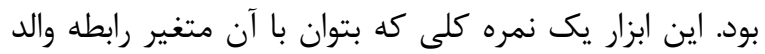

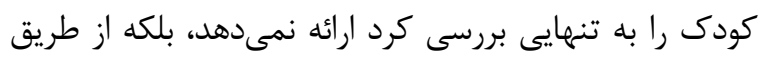

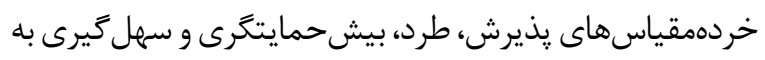

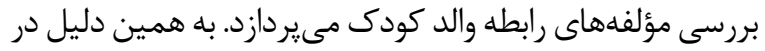

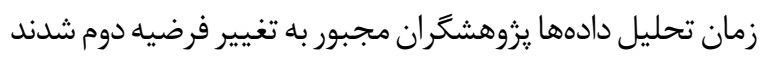

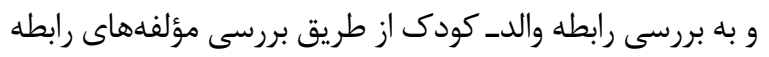

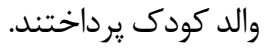

طبق اين محدوديتها جهت غناى نتايج يزوهشهاى آنتى آتى

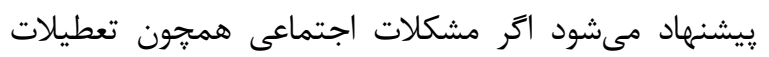

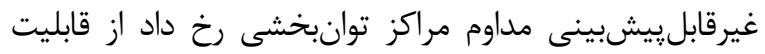

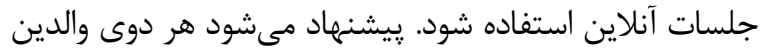

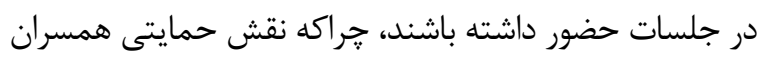

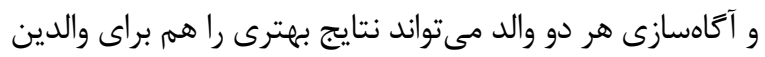

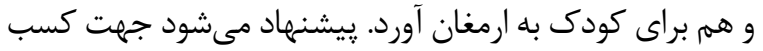

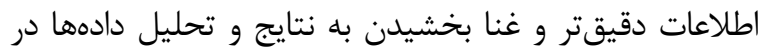

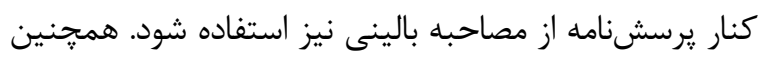

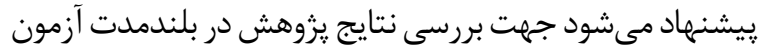

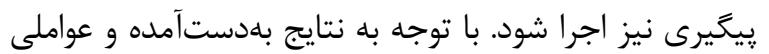

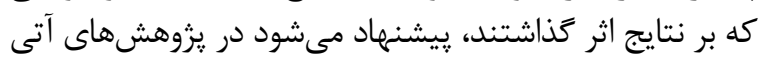

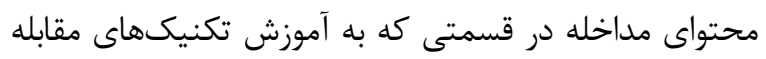

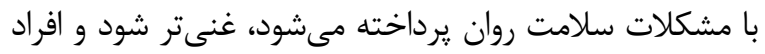

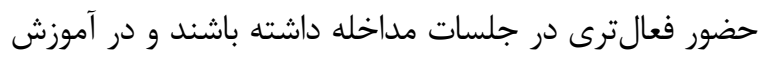

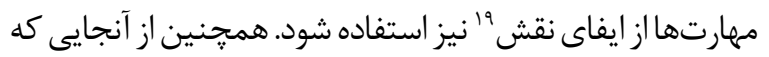

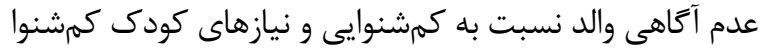

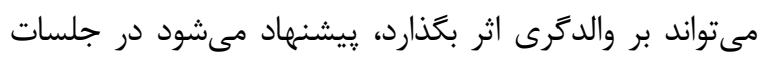

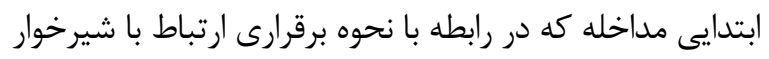

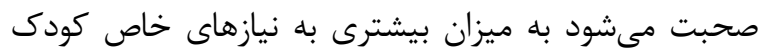

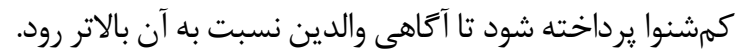

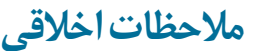

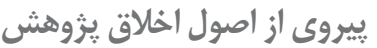

به منظور رعايت اصول اخلاقى مادرانى كه در يزوهش حضور

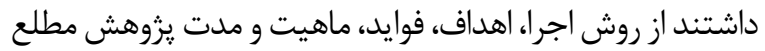

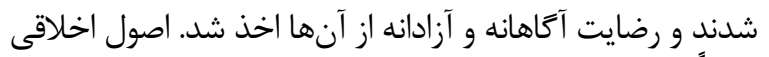

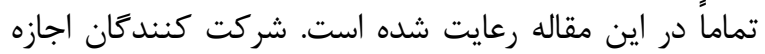

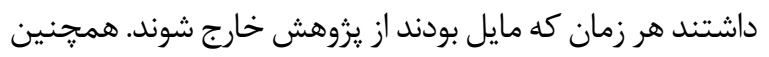




\section{References}

[1] World Health Organization. Deafness and hearing loss [Internet]. 2021 [Updated 2021 April 1]. Available from: https://www.who.int/ news-room/fact-sheets/detail/deafness-and-hearing-loss

[2] Harris C, Hemer S, Chur-Hansen A. Emotion as motivator: Parents, professionals and diagnosing childhood deafness. Medical Anthropology. 2021; 40(3):254-66. [DOI:10.1080/01459740.2020.1796659]

[3] Marriage J, Brown TH, Austin N. Hearing impairment in children. Paediatrics and Child Health. 2017; 27(10):441-6. [DOI:10.1016/j. paed.2017.06.003]

[4] Chaudhury S. Anxiety and depression in mothers of deaf children: Awareness needed. Medical Journal of Dr DY Patil University. 2014; 7(6):720-1. https://www.mjdrdypu.org/text. asp?2014/7/6/720/144856

[5] Green VA. Parental reaction to diagnosis of infant hearing loss. Advances in Mental Health. 2020; 18(1):62-72. [DOI:10.1080/18 387357.2019.1651661]

[6] Majorano M, Guerzoni L, Cuda D, Morelli M. Mothers' emotional experiences related to their child's diagnosis of deafness and cochlear implant surgery: Parenting stress and child's language development. International Journal of Pediatric Otorhinolaryngology. 2020; 130:109812. [DOI:10.1016/j.ijporl.2019.109812]

[7] Kobosko J, Geremek-Samsonowicz A, Skarżyński H. [Mental health problems of mothers and fathers of the deaf children with cochlear implants (Polish)]. Otolaryngologia Polska. 2014; 68(3):135-42. [DOI:10.1016/j.otpol.2013.05.005] [PMID]

[8] Saki N, Yadollahpour A, Moniri S, Karimi M, Bayat A, Abshirini $\mathrm{H}$, et al. Investigating the impacts of cochlear implantation on the happiness and self-esteem of mothers of children with severe hearing loss. International Journal of Mental Health and Addiction. 2017; 15(2):288-94. [DOI:10.1007/s11469-016-9672-4]

[9] Yamaoka Y, Tamiya N, Izumida N, Kawamura A, Takahashi H, Noguchi $\mathrm{H}$. The relationship between raising a child with a disability and the mental health of mothers compared to raising a child without disability in Japan. SSM - Population Health. 2016; 2:542-8. [DOI:10.1016/j.ssmph.2016.08.001]

[10] Gilson KM, Davis E, Johnson S, Gains J, Reddihough D, Williams $\mathrm{K}$. Mental health care needs and preferences for mothers of children with a disability. Child: Care, Health and Development. 2018; 44(3):384-91. [DOI:10.1111/cch.12556]

[11] Jean YQ, Mazlan R, Ahmad M, Maamor N. Parenting stress and maternal coherence: Mothers with deaf or hard-of-hearing children. American Journal of Audiology. 2018; 27(3):260-71. [DOI:10.1044/2018_AJA-17-0093]

[12] Faramarzi S. Comparing the quality of life and psychological wellbeing in mothers of children with hearing loss and mothers of children with other special needs. Auditory and Vestibular Research. 2017; 26(2):86-92. https://avr.tums.ac.ir/index.php/avr/article/ view/151

[13] Aras I, Stevanović R, Vlahović S, Stevanović S, Kolarić B, Kondić L. Health related quality of life in parents of children with speech and hearing impairment. International Journal of Pediatric Otorhinolaryngology. 2014; 78(2):323-9. [DOI:10.1016/j. ijporl.2013.12.001]
[14] Vänskä M, Punamäki RL, Lindblom J, Flykt M, Tolvanen A Unkila-Kallio L, et al. Parental pre- and postpartum mental health predicts child mental health and development. Family Relations. 2017; 66(3):497-511. [DOI:10.1111/fare.12260]

[15] Vallotton C, Harewood T, Froyen L, Brophy-Herb H, Ayoub C. Child behavior problems: Mothers' and fathers' mental health matters today and tomorrow. Early Childhood Research Quarterly. 2016; 37:81-93. [DOI:10.1016/j.ecresq.2016.02.006]

[16] Levin KA, Dallago L, Currie C. The association between adolescent life satisfaction, family structure, family affluence and gender differences in parent-child communication. Social Indicators Research. 2012; 106(2):287-305. [DOI:10.1007/s11205-011-9804-y]

[17] Kelley ML, Bravo AJ, Hamrick HC, Braitman AL, White TD, Jenkins J. Parents' reports of children's internalizing symptoms: Associations with parents' mental health symptoms and substance use disorder. Journal of Child and Family Studies. 2017; 26(6):1646-54. [DOI:10.1007/s10826-017-0677-9]

[18] Branje SJ, Hale III WW, Frijns T, Meeus WH. Longitudinal associations between perceived parent-child relationship quality and depressive symptoms in adolescence. Journal of Abnormal Child Psychology. 2010; 38(6):751-63. [DOI:10.1007/s10802010-9401-6]

[19] Yan J, Feng X, Schoppe-Sullivan SJ. Longitudinal associations between parent-child relationships in middle childhood and child-perceived loneliness. Journal of Family Psychology. 2018; 32(6):841-7. [DOI:10.1037/fam0000446]

[20] Schiff M, Pat-Horenczyk R, Ziv Y, Brom D. Multiple traumas, maternal depression, mother-child relationship, social support, and young children's behavioral problems. Journal of Interpersonal Violence. 2021; 36(1-2):892-914. [DOI:10.1177/0886260517725738]

[21] Nomaguchi K, Allen A. Mother-child relationship quality from preschool to adolescence: Variation by maternal education [Internet]. 2020 [Updated 2020 January 6]. Available from: https:// www.bgsu.edu/content/dam/BGSU/college-of-arts-and-sciences/center-for-family-and-demographic-research/documents/ working-papers/2019/WP-2019-09-Nomaguchi-Allen-MotherChild-Relationship-v2.pdf

[22] Giannotta F, Rydell AM. The role of the mother-child relationship in the route from child ADHD to adolescent symptoms of depressed mood. Journal of Adolescence. 2017; 61:40-9. [DOI:10.1016/j.adolescence.2017.09.005]

[23] Blank A, Frush Holt R, Pisoni DB, Kronenberger WG. Associations between parenting stress, language comprehension, and inhibitory control in children with hearing loss. Journal of Speech, Language, and Hearing Research. 2020; 63(1):321-33. [DOI:10.1044/2019_JSLHR-19-00230]

[24] Bahadori Khosroshahi J. [Communication parental self-efficacy and self forgiveness with mother-child interaction in mothers of children with mental retardation (Persian)]. Empowering Exceptional Children. 2017; 8(22):26-36. http://www.ceciranj.ir/article_63557.htm

[25] Antonopoulou K, Hadjikakou K, Stampoltzis A, Nicolaou N Parenting styles of mothers with deaf or hard-of-hearing children and hearing siblings. The Journal of Deaf Studies and Deaf Education. 2012; 17(3):306-18. [DOI:10.1093/deafed/ens013] 
[26] Howe D. Disabled children, parent-child interaction and attachment. Child \& Family Social Work. 2006; 11(2):95-106. [DOI:10.1111/j.1365-2206.2006.00397.x]

[27] Pinquart M. Do the parent-child relationship and parenting behaviors differ between families with a child with and without chronic illness? A meta-analysis. Journal of Pediatric Psychology. 2013; 38(7):708-21. [DOI:10.1093/jpepsy/jst020]

[28] Kurtzer-White E, Luterman D. Families and children with hearing loss: Grief and coping. Mental Retardation and Developmental Disabilities Research Reviews. 2003; 9(4):232-5. [DOI:10.1002/mrdd.10085]

[29] Janjua F, Woll B, Kyle J. Effects of parental style of interaction on language development in very young severe and profound deaf children. International Journal of Pediatric Otorhinolaryngology. 2002; 64(3):193-205. [DOI:10.1016/ S0165-5876(02)00069-1]

[30] Barker DH, Quittner AL, Fink NE, Eisenberg LS, Tobey EA, Niparko JK. Predicting behavior problems in deaf and hearing children: The influences of language, attention, and parent-child communication. Development and Psychopathology. 2009; 21(2):373-92. [DOI:10.1017/S0954579409000212]

[31] Muse C, Harrison J, Yoshinaga-Itano Ch, Grimes A, Brookhouser PE, Epstein S, et al. Supplement to the JCIH 2007 position statement: Principles and guidelines for early intervention after confirmation that a child is deaf or hard of hearing. Pediatrics. 2013; 131(4):e1324-49. [DOI:10.1542/peds.2013-0008]

[32] Ingber S, Most T. 38. Parental involvement in early intervention for children with hearing loss. In: Dattner E, Bar-On A, Ravid D, editors. Handbook of Communication Disorders: Theoretical, Empirical, and Applied Linguistic Perspectives. Handbooks of Applied Linguistics (HAL). Vol. 15. Berlin/Boston: De Gruyter Mouton; 2018. pp. 793-810. [DOI:10.1515/9781614514909039]

[33] Hitchins ARC, Hogan SC. Outcomes of early intervention for deaf children with additional needs following an Auditory Verbal approach to communication. International Journal of Pediatric Otorhinolaryngology. 2018; 115:125-32. [DOI:10.1016/j. ijporl.2018.09.025]

[34] Stewart V, Slattery M, McKee J. Deaf and hard of hearing early intervention: Perceptions of family-centered practice. Journal of Early Intervention. 2021; 43(3):221-34. [DOI:10.1177/1053815120962547]

[35] Nikkhooo F, Hassanzadeh S, Afrooz GA, Farzad V. [Early hearing, language, and attachment based interventions for deaf children under age of two (Persian)]. Journal of Paramedical Sciences \& Rehabilitation. 2018; 7(1):57-68. [DOI:10.22038/ JPSR.2018.20011.1500]

[36] Erbasi E, Scarinci N, Hickson L, Ching TYC. Parental involvement in the care and intervention of children with hearing loss. International Journal of Audiology. 2018; 57(Suppl 2):S15-26. [DOI:10.1080/14992027.2016.1220679]

[37] Jackson CW, Traub RJ, Turnbull AP. Parents' experiences with childhood deafness: Implications for family-centered services. Communication Disorders Quarterly. 2008; 29(2):82-98. [DOI:10.1177/1525740108314865]
[38] Shekari E, Nakhshab M, Valinejad V, Modarres Zadeh A, Hosseinpour AH. A systematic review of the effectiveness of early intervention and the role of parents in language development of hearing loss children. Iranian Rehabilitation Journal. 2017; 15(1):5-14. [DOI:10.18869/nrip.irj.15.1.5]

[39] Zaidman-Zait A, Most T, Tarrasch R, Haddad E. Mothers' and fathers' involvement in intervention programs for deaf and hard of hearing children. Disability and Rehabilitation. 2018; 40(11):1301-9. [DOI:10.1080/09638288.2017.1297491]

[40] Kulkarni KA, Gathoo VS. Parent empowerment in early intervention programmes of children with hearing loss in Mumbai, India. Disability, CBR \& Inclusive Development. 2017; 28(2):4558. [DOI:10.5463/dcid.v28i2.550]

[41] Wittkowski A, Cartwright K, Emsley R, Bee P, Camacho E, Calam R, et al. Enhancing maternal and infant wellbeing: study protocol for a feasibility trial of the Baby Triple P Positive Parenting programme for mothers with severe mental health difficulties (the IMAGINE study). Trials. 2018; 19:516. [DOI:10.1186/ s13063-018-2918-7]

[42] Tsivos ZL, Calam R, Sanders MR, Wittkowski A. A pilot randomised controlled trial to evaluate the feasibility and acceptability of the Baby Triple P Positive Parenting Programme in mothers with postnatal depression. Clinical Child Psychology and Psychiatry. 2015; 20(4):532-54. [DOI:10.1177/1359104514531589] [PMID] [PMCID]

[43] Butler H, Hare D, Walker S, Wieck A, Wittkowski A. The acceptability and feasibility of the Baby Triple P Positive Parenting Programme on a mother and baby unit: Q-methodology with mothers with severe mental illness. Archives of Women's Mental Health. 2014; 17(5):455-63. [DOI:10.1007/s00737-014-0429-4]

[44] Evans T, Boyd RN, Colditz P, Sanders M, Whittingham K. Baby Triple $P$ for parents of a very preterm infant: A case study. Journal of Child and Family Studies. 2017; 26(2):633-42. [DOI:10.1007/ s10826-016-0571-x]

[45] Popp L, Fuths S, Schneider S. The relevance of infant outcome measures: A pilot-RCT comparing Baby Triple P Positive Parenting Program with care as usual. Frontiers in Psychology. 2019; 10:2425. [DOI:10.3389/fpsyg.2019.02425]

[46] Evans T, Boyd RN, Colditz P, Sanders M, Whittingham K. Mother-very preterm infant relationship quality: RCT of Baby Triple P. Journal of Child and Family Studies. 2017; 26(1):284-95. [DOI:10.1007/s10826-016-0555-x]

[47] Sreekumar S, L RK, Nair LS, Joseph JM. Psychosocial concerns among parents of children with hearing impairment. Loyola Journal of Social Sciences. 2019;33(2):21-38. https://web.b.ebscohost. $\mathrm{com} /$ ehost $/$ pdfviewer $/$ pdfviewer?vid $=1 \&$ sid $=9 \mathrm{~d} 83720 \mathrm{e}-508 \mathrm{~b}-$ 4bac-9db3-57215609610f\%40sessionmgr101

[48] Chang CW, Biegel DE. Factors affecting mental health service utilization among Latino Americans with mental health issues. Journal of Mental Health. 2018; 27(6):552-9. [DOI:10.1080/09 638237.2017.1385742]

[49] Kiely KM, Leach LS, Olesen SC, Butterworth P. How financial hardship is associated with the onset of mental health problems over time. Social Psychiatry and Psychiatric Epidemiology. 2015; 50(6):909-18. [DOI:10.1007/s00127-015-1027-0] 
[50] Grandpierre V, Fitzpatrick EM, Thomas R, Mendonca O, Sikora L, Potter BK. Barriers and facilitators to cultural competence in early hearing loss services: A qualitative analysis. Canadian Journal of Speech-Language Pathology \& Audiology. 2019; 43(3):189-202. https://www.cjslpa.ca/detail.php?ID=1250\&lang=en

[51] Fitzpatrick EM, Gaboury I, Durieux-Smith A, Coyle D, Whittingham JA, Nassrallah F. Auditory and language outcomes in children with unilateral hearing loss. Hearing Research. 2019; 372:42-51. [DOI:10.1016/j.heares.2018.03.015]

[52] Ebrahimi H, Mohammadi E, Mohammadi MA, Pirzadeh A, Mahmoudi H, Ansari I. Stigma in mothers of deaf children. Iranian Journal of Otorhinolaryngology. 2015; 27(2):109-18. [DOI:10.22038/IJORL.2015.3614]

[53] Åsberg KK, Vogel JJ, Bowers CA. Exploring correlates and predictors of stress in parents of children who are deaf: Implications of perceived social support and mode of communication. Journal of Child and Family Studies. 2008; 17(4):486-99. [DOI:10.1007/ s10826-007-9169-7]

[54] Prakash SS, Prakash SGR, Ravichandran A, Susan KY, Alex W. Measuring levels of stress and depression in mothers of children using hearing aids and cochlear implants: A comparative study. International Journal of Special Education. 2013; 28(1):37-44. https://eric.ed.gov/?id=EJ1013685

[55] de Diego-Lázaro B, Restrepo MA, Sedey AL, Yoshinaga-Itano Ch. Predictors of vocabulary outcomes in children who are deaf or hard of hearing from Spanish-speaking families. Language, Speech, and Hearing Services in Schools. 2019; 50(1):113-25. [DOI:10.1044/2018_LSHSS-17-0148]

[56] Sahli S. Investigating child raising attitudes of fathers having or not having a child with hearing loss. International Journal of Pediatric Otorhinolaryngology. 2011; 75(5):681-5. [DOI:10.1016/j. ijporl.2011.02.013]

[57] Merugumala SV, Pothula V, Cooper M. Barriers to timely diagnosis and treatment for children with hearing impairment in a Southern Indian city: A qualitative study of parents and clinic staff. International Journal of Audiology. 2017; 56(10):733-9. [DOI:10.1 080/14992027.2017.1340678] 
This Page Intentionally Left Blank 